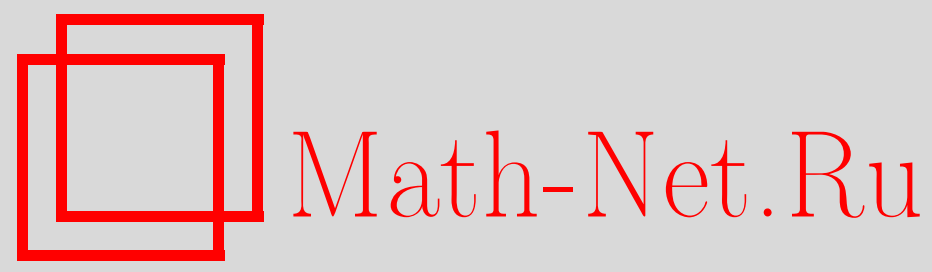

М. К. Потапов, Б. В. Симонов, С. Ю. Тихонов, Преобразование рядов Фурье с помощью степенных и слабо колеблющихся последовательностей, Матем. заметки, 2005, том 77, выпуск 1, 99-116

DOI: https://doi.org/10.4213/mzm2473

Использование Общероссийского математического портала Math-Net.Ru подразумевает, что вы прочитали и согласны с пользовательским соглашением http://www . mathnet.ru/rus/agreement

Параметры загрузки:

IP : 3.95 .254 .165

26 апреля 2023 г., 13:10:42

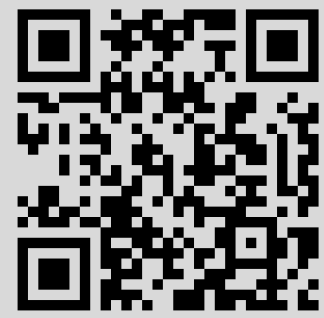




\title{
ПРЕОБРАЗОВАНИЕ РЯДОВ ФУРЬЕ С ПОМОЩЬЮ СТЕПЕННЫХ И СЛАБО КОЛЕБЛЮЩИХСЯ ПОСЛЕДОВАТЕЛЬНОСТЕЙ
}

\author{
М.К. Потапов, Б. В. Симонов, С. Ю. Тихонов
}

В данной работе рассматриваются преобразования рядов Фурье функций многих переменных с помощью произведения степенных и слабо колеблющихся последовательностей. Получены оценки смешанных модулей гладкости преобразованных рядов Фурье через смешанные модули гладкости самих функций.

Библиографофия: 9 названий.

1. Введение. Пусть $L_{p}^{0}, 1<p<\infty,-$ пространство всех измеримых функций двух переменных $f=f\left(x_{1}, x_{2}\right), 2 \pi$-периодических по каждой переменной, для которых

$$
\begin{gathered}
\|f\|_{p}=\left(\int_{0}^{2 \pi} \int_{0}^{2 \pi}\left|f\left(x_{1}, x_{2}\right)\right|^{p} d x_{1} d x_{2}\right)^{1 / p}<\infty, \\
\int_{0}^{2 \pi} f\left(x_{1}, x_{2}\right) d x_{1}=0 \quad \text { для почти всех } x_{2}, \\
\int_{0}^{2 \pi} f\left(x_{1}, x_{2}\right) d x_{2}=0 \quad \text { для почти всех } x_{1} ;
\end{gathered}
$$

$\omega_{\beta_{1}, \beta_{2}}\left(f, \delta_{1}, \delta_{2}\right)_{p}$ - смешанный модуль гладкости функции $f$ (в метрике $L_{p}$ ) порядков $\beta_{1}$ и $\beta_{2}\left(\beta_{1}, \beta_{2}>0\right)$ соответственно по переменным $x_{1}$ и $x_{2}$, т.е.

$$
\omega_{\beta_{1}, \beta_{2}}\left(f, \delta_{1}, \delta_{2}\right)_{p}=\sup _{\left|h_{1}\right| \leqslant \delta_{1},\left|h_{2}\right| \leqslant \delta_{2}}\left\|\Delta_{h_{1}}^{\beta_{1}}\left(\Delta_{h_{2}}^{\beta_{2}} f\right)\right\|_{p},
$$

где

$$
\begin{gathered}
\Delta_{h_{1}}^{\beta_{1}} f\left(x_{1}, x_{2}\right)=\sum_{\nu_{1}=0}^{\infty}(-1)^{\nu_{1}}\left(\begin{array}{l}
\beta_{1} \\
\nu_{1}
\end{array}\right) f\left(x_{1}+\left(\beta_{1}-\nu_{1}\right) h_{1}, x_{2}\right), \\
\Delta_{h_{2}}^{\beta_{2}} f\left(x_{1}, x_{2}\right)=\sum_{\nu_{2}=0}^{\infty}(-1)^{\nu_{2}}\left(\begin{array}{l}
\beta_{2} \\
\nu_{2}
\end{array}\right) f\left(x_{1}, x_{2}+\left(\beta_{2}-\nu_{2}\right) h_{2}\right), \\
\left(\begin{array}{c}
\beta_{i} \\
\nu_{i}
\end{array}\right)=\frac{\beta_{i}\left(\beta_{i}-1\right) \cdots\left(\beta_{i}-\nu_{i}+1\right)}{\nu_{i} !} \quad \text { для } \nu_{i} \geqslant 1, \quad\left(\begin{array}{c}
\beta_{i} \\
0
\end{array}\right)=1, \quad i=1,2 .
\end{gathered}
$$

Работа выполнена при поддержке Российского фонда фундаментальных исследований, гранты № 03-01-00080 и № 03-01-06155, и программы поддержки ведущих научных школ, грант №HШ1657.2003 .1$. 
Ряд Фурье функции $f \in L_{p}^{0}$ может быть записан в виде

$$
\begin{aligned}
& \sum_{n_{1}=1}^{\infty} \sum_{n_{2}=1}^{\infty}\left(a_{n_{1} n_{2}}^{(1)} \cos n_{1} x_{1} \cos n_{2} x_{2}+a_{n_{1} n_{2}}^{(2)} \sin n_{1} x_{1} \cos n_{2} x_{2}\right. \\
& \left.\quad+a_{n_{1} n_{2}}^{(3)} \cos n_{1} x_{1} \sin n_{2} x_{2}+a_{n_{1} n_{2}}^{(4)} \sin n_{1} x_{1} \sin n_{2} x_{2}\right) \equiv \sum_{n_{1}=1}^{\infty} \sum_{n_{2}=1}^{\infty} A_{n_{1} n_{2}}\left(x_{1}, x_{2}\right) .
\end{aligned}
$$

Пусть дана последовательность чисел $\lambda=\left\{\lambda_{n_{1} n_{2}}\right\}_{n_{1}, n_{2} \in \mathbb{N}}$. Преобразованным рядом Фурье для ряда (1) назовем ряд

$$
\sigma(\lambda, f)=\sum_{n_{1}=1}^{\infty} \sum_{n_{2}=1}^{\infty} \lambda_{n_{1} n_{2}} A_{n_{1} n_{2}}\left(x_{1}, x_{2}\right) .
$$

Если последовательность $\lambda$ степенного типа: $\lambda_{n_{1} n_{2}}=n_{1}^{\sigma_{1}} n_{2}^{\sigma_{2}}$, где $\sigma_{i}>0$ для $i=1,2$, то ряд (2) можно считать (см. [1]) рядом Фурье смешанной производной $f^{\left(\sigma_{1}, \sigma_{2}\right)}$ функции $f \in L_{p}^{0}$.

Пусть даны положительные числа $r_{1}, r_{2}, \beta_{1}, \beta_{2}, \alpha_{1}, \alpha_{2}$. В работе [2] получены оценки смешанного модуля гладкости порядков $\beta_{1}$ и $\beta_{2}$ производной $f^{\left(\sigma_{1}, \sigma_{2}\right)}$ через смешанный модуль гладкости порядков $r_{1}+\beta_{1}$ и $r_{2}+\beta_{2}$ функции $f$ в следующих двух случаях:

I. $\sigma_{1}=r_{1}, \sigma_{2}=r_{2}$;

II. $\sigma_{1}=r_{1}-\alpha_{1}, \sigma_{2}=r_{2}-\alpha_{2}$.

Там же было показано, что в случае

III. $\sigma_{1}=r_{1}+\alpha_{1}, \sigma_{2}=r_{2}+\alpha_{2}$

уже невозможно получить аналогичные двусторонние оценки смешанного модуля гладкости, но получены двусторонние оценки интегральных средних смешанного модуля гладкости.

Возникает вопрос, как изменятся оценки типов II и III, если вместо последовательностей степенного порядка $\lambda_{n_{1} n_{2}}=n_{1}^{r_{1} \mp \alpha_{1}} n_{2}^{r_{2} \mp \alpha_{2}}$ рассмотреть последовательности степенного и логарифмического порядка:

$$
\begin{aligned}
& \text { IV. } \lambda_{n_{1} n_{2}}=\frac{n_{1}^{r_{1}}}{\ln _{k_{1}}^{A_{1}}\left(d_{k_{1}} n_{1}\right)} \frac{n_{2}^{r_{2}}}{\ln _{k_{2}}^{A_{2}}\left(d_{k_{2}} n_{2}\right)} ; \\
& \text { V. } \lambda_{n_{1} n_{2}}=n_{1}^{r_{1}} \ln _{k_{1}}^{A_{1}}\left(d_{k_{1}} n_{1}\right) n_{2}^{r_{2}} \ln _{k_{2}}^{A_{2}}\left(d_{k_{2}} n_{2}\right) .
\end{aligned}
$$

Здесь $A_{j}>0, k_{j} \in \mathbb{N}$ и $\ln _{1} u=\ln u, \ln _{i} u=\ln \left(\ln _{i-1} u\right), i=2,3, \ldots, k_{j}$, а константы $d_{k_{j}}$ удовлетворяют условиям $\ln _{k_{j}} d_{k_{j}}>1, j=1,2$.

В работе [3] дан ответ на этот вопрос для случая IV. В данной работе получен ответ для случая V. Он выглядит следующим образом.

УТВЕРЖДЕНИЕ. Пусть числа $p, \tau, \theta, \beta, r$ таковы, что $1<p<\infty, 0<\theta \leqslant$ $\min (2, p), \max (2, p) \leqslant \tau<\infty, \beta_{j}, r_{j}, A_{j}>0, k_{j} \in \mathbb{N}, j=1,2$, и при әтом константыл $d_{s}$ удовлетворяют условиям $\ln _{s} d_{s}>1, s=1, \ldots, \max \left(k_{1}, k_{2}\right)$.

а) Если для функиии $f \in L_{p}^{0}$ выполнено условие

$$
\begin{aligned}
J(f, \theta, p):= & \left(\int_{0}^{1} \int_{0}^{1} t_{1}^{-r_{1} \theta-1} t_{2}^{-r_{2} \theta-1} \ln _{k_{1}}^{A_{1}}\left(\frac{d_{k_{1}}}{t_{1}}\right) \ln _{k_{2}}^{A_{2}}\left(\frac{d_{k_{2}}}{t_{2}}\right)\right. \\
& \left.\times \omega_{r_{1}+\beta_{1}, r_{2}+\beta_{2}}^{\theta}\left(f, t_{1}, t_{2}\right)_{p} d t_{1} d t_{2}\right)^{1 / \theta}<\infty,
\end{aligned}
$$


то существует функиия $\varphi \in L_{p}^{0}$, имеющая ряд Фурье $\sigma(\lambda, f)$, где

$$
\lambda=\left\{\lambda_{n_{1} n_{2}}=n_{1}^{r_{1}} n_{2}^{r_{2}} \ln _{k_{1}}^{A_{1}}\left(d_{k_{1}} n_{1}\right) \ln _{k_{2}}^{A_{2}}\left(d_{k_{2}} n_{2}\right)\right\},
$$

причем $\|\varphi\|_{p} \leqslant C_{1} J(f, \theta, p)$ и для любых $\delta_{1}, \delta_{2} \in(0,1 / 2]$ справедливо неравенство $D\left(\varphi, \delta_{1}, \delta_{2}, \tau, p\right) \leqslant C_{2} H\left(f, \delta_{1}, \delta_{2}, \theta, p\right)$, где

$$
\begin{aligned}
& D\left(\varphi, \delta_{1}, \delta_{2}, \tau, p\right):=\left\{\delta_{1}^{\beta_{1} \tau} \ln _{k_{1}}^{A_{1}}\left(\frac{d_{k_{1}}}{\delta_{1}}\right) \int_{\delta_{1}}^{1} \frac{t_{1}^{-\beta_{1} \tau-1}}{\prod_{i=1}^{k_{1}} \ln _{i} \frac{d_{i}}{t_{1}} \ln _{k_{1}}^{A_{1} \tau} \frac{d_{k_{1}}}{t_{1}}} \omega_{\beta_{1} \beta_{2}}^{\tau}\left(\varphi, t_{1}, \delta_{2}\right)_{p} d t_{1}\right. \\
& +\delta_{2}^{\beta_{2} \tau} \ln _{k_{2}}^{A_{2}}\left(\frac{d_{k_{2}}}{\delta_{2}}\right) \int_{\delta_{2}}^{1} \frac{t_{2}^{-\beta_{2} \tau-1}}{\prod_{i=1}^{k_{2}} \ln _{i} \frac{d_{i}}{t_{2}} \ln _{k_{2}}^{A_{2} \tau} \frac{d_{k_{2}}}{t_{2}}} \omega_{\beta_{1} \beta_{2}}^{\tau}\left(\varphi, \delta_{1}, t_{2}\right)_{p} d t_{2}+\omega_{\beta_{1} \beta_{2}}^{\tau}\left(\varphi, \delta_{1}, \delta_{2}\right)_{p} \\
& +\delta_{1}^{\beta_{1} \tau} \delta_{2}^{\beta_{2} \tau} \ln _{k_{1}}^{A_{1}}\left(\frac{d_{k_{1}}}{\delta_{1}}\right) \ln _{k_{2}}^{A_{2}}\left(\frac{d_{k_{2}}}{\delta_{2}}\right) \\
& \left.\quad \times \int_{\delta_{1}}^{1} \int_{\delta_{2}}^{1} \frac{t_{1}^{-\beta_{1} \tau-1} t_{2}^{-\beta_{2} \tau-1}}{\prod_{i=1}^{k_{1}} \ln _{i} \frac{d_{i}}{t_{1}} \ln _{k_{1}}^{A_{1} \tau} \frac{d_{k_{1}}}{t_{1}} \prod_{i=1}^{k_{2}} \ln _{i} \frac{d_{i}}{t_{2}} \ln _{k_{2}}^{A_{2} \tau} \frac{d_{k_{2}}}{t_{2}}} \omega_{\beta_{1} \beta_{2}}^{\tau}\left(\varphi, t_{1}, t_{2}\right)_{p} d t_{1} d t_{2}\right\}^{1 / \tau} \\
& H\left(f, \delta_{1}, \delta_{2}, \theta, p\right):=\left\{\int_{0}^{\delta_{1}} \int_{0}^{\delta_{2}} t_{1}^{-r_{1} \theta-1} t_{2}^{-r_{2} \theta-1} \ln _{k_{1}}^{A_{1}}\left(\frac{d_{k_{1}}}{t_{1}}\right) \ln _{k_{2}}^{A_{2}}\left(\frac{d_{k_{2}}}{t_{2}}\right)\right. \\
& \left.\quad \times \omega_{r_{1}+\beta_{1}, r_{2}+\beta_{2}}^{\theta}\left(f, t_{1}, t_{2}\right)_{p} d t_{1} d t_{2}\right\}^{1 / \theta}
\end{aligned}
$$

и константы $C_{1}$ и $C_{2}$ не зависят от $f, \delta_{1}, \delta_{2}$.

b) Если для функиии $f \in L_{p}^{0}$ существует функиия $\varphi \in L_{p}^{0}$ с рядом Фурье $\sigma(\lambda, f)$, где $\lambda=\left\{\lambda_{n_{1} n_{2}}=n_{1}^{r_{1}} n_{2}^{r_{2}} \ln _{k_{1}}^{A_{1}}\left(d_{k_{1}} n_{1}\right) \ln _{k_{2}}^{A_{2}}\left(d_{k_{2}} n_{2}\right)\right\}$, mo $J(f, \tau, p) \leqslant C_{3}\|\varphi\|_{p}$ и для любых $\delta_{1}, \delta_{2} \in(0,1 / 2]$ справедливо неравенство $H\left(f, \delta_{1}, \delta_{2}, \tau, p\right) \leqslant C_{4} D\left(\varphi, \delta_{1}, \delta_{2}, \theta, p\right)$, где константы $C_{3}$ и $C_{4}$ не зависят от $f, \delta_{1}, \delta_{2}$.

Это утверждение следует из доказанной ниже теоремы 1.

2. Определения и обозначения. Пусть функция $f\left(x_{1}, x_{2}\right) \in L_{p}^{0}$ имеет ряд Фурье (1). Обозначим $b_{n_{1} n_{2}}:=a_{n_{1} n_{2}}^{(1)}+a_{n_{1} n_{2}}^{(2)}+a_{n_{1} n_{2}}^{(3)}+a_{n_{1} n_{2}}^{(4)}, n_{1}, n_{2}=1,2, \ldots$,

$$
\Delta_{m_{1} m_{2}}:=\sum_{n_{1}=\left[2^{m_{1}-2}\right]+1}^{2^{m_{1}-1}} \sum_{n_{2}=\left[2^{m_{2}-2}\right]+1}^{2^{m_{2}-1}} A_{n_{1} n_{2}}\left(x_{1}, x_{2}\right), \quad m_{1}, m_{2}=1,2, \ldots
$$

где $\left[2^{m_{2}-2}\right]=0$ для $m_{i}=1$ и $\left[2^{m_{i}-2}\right]=2^{m_{i}-2}$ для $m_{i}=2,3, \ldots, i=1,2$.

Пусть $Q M$ - класс функций $f \in L_{p}^{0}$, коэффициенты Фурье которых неотрицательны и удовлетворяют условиям: сушествуют неотрицательные числа $\gamma_{1}$ и $\gamma_{2}$ такие, что $\left(i=1,2,3,4, n_{1}, n_{2}=1,2, \ldots\right)$

$$
\begin{aligned}
& \Delta_{\gamma_{1}}^{(1,0)}\left(a_{n_{1} n_{2}}^{(i)}\right)=a_{n_{1} n_{2}}^{(i)} n_{1}^{-\gamma_{1}}-a_{n_{1}+1, n_{2}}^{(i)}\left(n_{1}+1\right)^{-\gamma_{1}} \geqslant 0, \\
& \Delta_{\gamma_{2}}^{(0,1)}\left(a_{n_{1} n_{2}}^{(i)}\right)=a_{n_{1} n_{2}}^{(i)} n_{2}^{-\gamma_{2}}-a_{n_{1}, n_{2}+1}^{(i)}\left(n_{2}+1\right)^{-\gamma_{2}} \geqslant 0, \\
& \Delta_{\gamma_{1} \gamma_{2}}^{(1,1)}\left(a_{n_{1} n_{2}}^{(i)}\right)=\Delta_{\gamma_{2}}^{(0,1)}\left(\Delta_{\gamma_{1}}^{(1,0)}\left(a_{n_{1} n_{2}}^{(i)}\right)\right) \geqslant 0 ;
\end{aligned}
$$


$\mathscr{L}$ - класс функций $f \in L_{p}^{0}$, коэффициенты Фурье которых неотрицательны и равны нулю, если $n_{1} \neq 2^{m_{1}}$ или если $n_{2} \neq 2^{m_{2}}, m_{1}, m_{2}=0,1,2, \ldots$

Если $F\left(f, \delta_{1}, \delta_{2}, s, \mu, \ldots\right) \geqslant 0$ и $G\left(f, \delta_{1}, \delta_{2}, s, \mu, \ldots\right) \geqslant 0$, то запись

$$
F\left(f, \delta_{1}, \delta_{2}, s, \mu, \ldots\right) \ll G\left(f, \delta_{1}, \delta_{2}, s, \mu, \ldots\right)
$$

будет означать, что существует такая постоянная $C$, не зависящая от $f, \delta_{1}, \delta_{2}$ и такая, что $F\left(f, \delta_{1}, \delta_{2}, s, \mu, \ldots\right) \leqslant C G\left(f, \delta_{1}, \delta_{2}, s, \mu, \ldots\right)$. Если одновременно

$$
F\left(f, \delta_{1}, \delta_{2}, s, \mu, \ldots\right) \ll G\left(f, \delta_{1}, \delta_{2}, s, \mu, \ldots\right)
$$

и

$$
G\left(f, \delta_{1}, \delta_{2}, s, \mu, \ldots\right) \ll F\left(f, \delta_{1}, \delta_{2}, s, \mu, \ldots\right),
$$

то будем писать

$$
G\left(f, \delta_{1}, \delta_{2}, s, \mu, \ldots\right) \asymp F\left(f, \delta_{1}, \delta_{2}, s, \mu, \ldots\right) .
$$

Функцию $\xi(t)$ будем назьвать слабо колеблющейся, если она измерима на $[D ;+\infty)$, $D>0$, положительна и для любого $\varkappa>0$ удовлетворяет соотношению

$$
\lim _{t \rightarrow \infty} \frac{\xi(\varkappa t)}{\xi(t)}=1
$$

Будем говорить (см. [4]), что слабо колеблющаяся функция $\mu(t)$ удовлетворяет условию $\left(S l_{s}\right)$ и обозначать $\mu(t) \in S l_{s}$, если выполнено условие

$$
\int_{0}^{1}\left[\mu\left(\frac{1}{t}\right)\right]^{-s} \frac{d t}{t}<\infty .
$$

Если $\mu(t)$ удовлетворяет условию $\left(S l_{s}\right)$, то для сокращения записи будем обозначать

$$
\gamma\left(\frac{1}{\delta}\right):=\left(\int_{0}^{\delta}\left[\mu\left(\frac{1}{t}\right)\right]^{-s} \frac{d t}{t}\right)^{-1 / s} .
$$

ПРИМЕР 1. Функция

$$
\mu(t)=\left(\prod_{i=1}^{k} \ln _{i} d_{i} t\right)^{1 / s} \ln _{k}^{A} d_{k} t, \quad t \in[1 ;+\infty),
$$

где $A, s>0$, а константы $d_{i}$ удовлетворяют условиям $\ln _{i} d_{i}>1, i=1, \ldots, k$, является слабо колеблющейся, $\mu(t) \in S l_{s}$, причем $\gamma(t) \asymp \ln _{k}^{A}\left(d_{k} t\right)$, т.е. последовательность $\gamma(n)$ эквивалентна логарифмической последовательности в случае $\mathrm{V}$.

Таким образом, преобразование рядов Фурье для случая V является частным случаем преобразований с помощью более общих последовательностей

$$
\lambda_{n_{1} n_{2}}=n_{1}^{r_{1}} \xi\left(n_{1}\right) n_{2}^{r_{2}} \xi\left(n_{2}\right)
$$

где $\xi(t)$ - возрастающая слабо колеблющаяся функция, и в дальнейшем мы будем рассматривать именно последовательности (4). 


\section{3. Основные теоремы.}

Teорема 1. Пусть $1<p<\infty, 0<\theta \leqslant \min (2, p), \max (2, p) \leqslant \tau<\infty, \beta_{i}, r_{i}>0 u$ функиии $\mu_{i}(t)$ слабо колеблющиеся, $i=1,2$.

а) $Е с л и \mu_{1}\left(t_{1}\right), \mu_{2}\left(t_{2}\right) \in S l_{\theta}$ и для функиии $f \in L_{p}^{0}$ выполнено условие

$$
\begin{aligned}
I(f, \theta, p):= & \left(\int_{0}^{1} \int_{0}^{1} t_{1}^{-r_{1} \theta-1} t_{2}^{-r_{2} \theta-1} \gamma_{1}^{\theta}\left(\frac{1}{t_{1}}\right) \gamma_{2}^{\theta}\left(\frac{1}{t_{2}}\right)\right. \\
& \left.\times \omega_{r_{1}+\beta_{1}, r_{2}+\beta_{2}}^{\theta}\left(f, t_{1}, t_{2}\right)_{p} d t_{1} d t_{2}\right)^{1 / \theta}<\infty
\end{aligned}
$$

то существует функиия $\varphi \in L_{p}^{0}$, имеющая ряд Фурве $\sigma(\lambda, f)$, әде $\lambda=\left\{\lambda_{n_{1} n_{2}}=\right.$ $\left.n_{1}^{r_{1}} n_{2}^{r_{2}} \gamma_{1}\left(n_{1}\right) \gamma_{2}\left(n_{2}\right)\right\}$, причем $\|\varphi\|_{p} \ll I(f, \theta, p)$ и для любых $\delta_{1}, \delta_{2} \in(0,1 / 2]$ справедливо неравенство $A\left(\varphi, \delta_{1}, \delta_{2}, \tau, p\right) \ll B\left(f, \delta_{1}, \delta_{2}, \theta, p\right)$, где

$$
\begin{aligned}
& A\left(\varphi, \delta_{1}, \delta_{2}, \tau, p\right):=\left\{\delta_{1}^{\beta_{1} \tau} \delta_{2}^{\beta_{2} \tau} \gamma_{1}^{\tau}\left(\frac{1}{\delta_{1}}\right) \gamma_{2}^{\tau}\left(\frac{1}{\delta_{2}}\right) \int_{\delta_{1}}^{1} \int_{\delta_{2}}^{1} \frac{t_{1}^{-\beta_{1} \tau-1}}{\mu_{1}^{\tau}\left(\frac{1}{t_{1}}\right)} \frac{t_{2}^{-\beta_{2} \tau-1}}{\mu_{2}^{\tau}\left(\frac{1}{t_{2}}\right)}\right. \\
& \times \omega_{\beta_{1} \beta_{2}}^{\tau}\left(\varphi, t_{1}, t_{2}\right)_{p} d t_{1} d t_{2} \\
&+ \delta_{1}^{\beta_{1} \tau} \gamma_{1}^{\tau}\left(\frac{1}{\delta_{1}}\right) \int_{\delta_{1}}^{1} \frac{t_{1}^{-\beta_{1} \tau-1}}{\mu_{1}^{\tau}\left(\frac{1}{t_{1}}\right)} \omega_{\beta_{1} \beta_{2}}^{\tau}\left(\varphi, t_{1}, \delta_{2}\right)_{p} d t_{1} \\
&+\left.\delta_{2}^{\beta_{2} \tau} \gamma_{2}^{\tau}\left(\frac{1}{\delta_{2}}\right) \int_{\delta_{2}}^{1} \frac{t_{2}^{-\beta_{2} \tau-1}}{\mu_{2}^{\tau}\left(\frac{1}{t_{2}}\right)} \omega_{\beta_{1} \beta_{2}}^{\tau}\left(\varphi, \delta_{1}, t_{2}\right)_{p} d t_{2}+\omega_{\beta_{1} \beta_{2}}^{\tau}\left(\varphi, \delta_{1}, \delta_{2}\right)_{p}\right\}^{1 / \tau}, \\
& B\left(f, \delta_{1}, \delta_{2}, \theta, p\right):=\left\{\int_{0}^{\delta_{1}} \int_{0}^{\delta_{2}} t_{1}^{-r_{1} \theta-1} t_{2}^{-r_{2} \theta-1} \gamma_{1}^{\theta}\left(\frac{1}{t_{1}}\right) \gamma_{2}^{\theta}\left(\frac{1}{t_{2}}\right)\right. \\
&\left.\quad \times \omega_{r_{1}+\beta_{1}, r_{2}+\beta_{2}}^{\theta}\left(f, t_{1}, t_{2}\right)_{p} d t_{1} d t_{2}\right\}^{1 / \theta} \cdot
\end{aligned}
$$

b) Если $\mu_{1}\left(t_{1}\right), \mu_{2}\left(t_{2}\right) \in S l_{\tau}$ и для функиии $f \in L_{p}^{0}$ существует функиия $\varphi \in L_{p}^{0}$ с рядом Фурье $\sigma(\lambda, f)$, где $\lambda=\left\{\lambda_{n_{1} n_{2}}=n_{1}^{r_{1}} n_{2}^{r_{2}} \gamma_{1}\left(n_{1}\right) \gamma_{2}\left(n_{2}\right)\right\}$, mo $I(f, \tau, p) \ll\|\varphi\|_{p} u$ для любых $\delta_{1}, \delta_{2} \in(0,1 / 2]$ справедливо неравенство $B\left(f, \delta_{1}, \delta_{2}, \tau, p\right) \ll A\left(\varphi, \delta_{1}, \delta_{2}, \theta, p\right)$.

ТЕорема 2. Если $f \in Q M$, то утверждения теоремы 1 справедливы при замене условий $0<\theta \leqslant \min (2, p)$ u $\max (2, p) \leqslant \tau<\infty$ на условия $0<\theta \leqslant p u$ $p \leqslant \tau<\infty$.

ТЕОрема 3. Если $f \in \mathscr{L}$, то утвержсдения теоремы 1 справедливы при замене условий $0<\theta \leqslant \min (2, p)$ u $\max (2, p) \leqslant \tau<\infty$ на условия $0<\theta \leqslant 2 u 2 \leqslant \tau<\infty$.

Следствия. Пусть $1<p<\infty, 0<\theta \leqslant \min (2, p), \max (2, p) \leqslant \tau<\infty, \beta_{i}, r_{i}>0 u$ функиии $\mu_{i}(t)$ слабо колеблюшиеся, $i=1,2$.

а) $Е с л и \mu_{1}\left(t_{1}\right), \mu_{2}\left(t_{2}\right) \in S l_{2}$ и функиия $f \in L_{2}^{0}$, то для существования функции $\varphi \in L_{2}^{0}$ с рядом Фурве $\sigma(\lambda, f)$, әде $\lambda=\left\{\lambda_{n_{1} n_{2}}=n_{1}^{r_{1}} n_{2}^{r_{2}} \gamma_{1}\left(n_{1}\right) \gamma_{2}\left(n_{2}\right)\right\}$, необходимо $u$ достаточно, чтобы $I(f, 2,2)<\infty$, причем для любых $\delta_{1}, \delta_{2} \in(0,1 / 2]$ справедливо соотношение $B\left(f, \delta_{1}, \delta_{2}, 2,2\right) \asymp A\left(\varphi, \delta_{1}, \delta_{2}, 2,2\right)$.

b) Ecли $\mu_{1}\left(t_{1}\right), \mu_{2}\left(t_{2}\right) \in S l_{p}$ и функиия $f \in L_{p}^{0} \cap Q M$, то для существования функиии $\varphi \in L_{p}^{0}$ с рядом Фурье $\sigma(\lambda, f)$, где $\lambda=\left\{\lambda_{n_{1} n_{2}}=n_{1}^{r_{1}} n_{2}^{r_{2}} \gamma_{1}\left(n_{1}\right) \gamma_{2}\left(n_{2}\right)\right\}$, 
необходимо и достаточно, чтобы $I(f, p, p)<\infty$, причем для любых $\delta_{1}, \delta_{2} \in(0,1 / 2]$ справедливо соотношение $B\left(f, \delta_{1}, \delta_{2}, p, p\right) \asymp A\left(\varphi, \delta_{1}, \delta_{2}, p, p\right)$.

c) Если $\mu_{1}\left(t_{1}\right), \mu_{2}\left(t_{2}\right) \in S l_{2}$ и функция $f \in L_{p}^{0} \cap \mathscr{L}$, то для существования функиии $\varphi \in L_{p}^{0}$ с рядом Фурье $\sigma(\lambda, f)$, где $\lambda=\left\{\lambda_{n_{1} n_{2}}=n_{1}^{r_{1}} n_{2}^{r_{2}} \gamma_{1}\left(n_{1}\right) \gamma_{2}\left(n_{2}\right)\right\}$, необходимо $u$ достаточно, чтобы $I(f, 2, p)<\infty$, причем для любых $\delta_{1}, \delta_{2} \in(0,1 / 2]$ справедливо соотношение $B\left(f, \delta_{1}, \delta_{2}, 2, p\right) \asymp A\left(\varphi, \delta_{1}, \delta_{2}, 2, p\right)$.

\section{4. Вспомогательные результаты.}

Лемма 1 (см. [5]). Пусть $1<p<\infty, 0<\theta \leqslant \min (2, p), \max (2, p) \leqslant \tau<\infty$, $\beta_{i}, r_{i}>0, i=1,2$.

а) Если для функиии $f \in L_{p}^{0}$ выполнено условие $I(f, \theta, p)<\infty$, то существует функиия $\psi \in L_{p}^{0}$, имеющая ряд Фурье $\sigma(\lambda, f)$, где $\lambda=\left\{\lambda_{n_{1} n_{2}}=n_{1}^{r_{1}} n_{2}^{r_{2}} \gamma_{1}\left(n_{1}\right) \gamma_{2}\left(n_{2}\right)\right\}$, причем $\|\psi\|_{p} \ll I(f, \theta, p)$.

b) Если для функиии $f \in L_{p}^{0}$ существует функиия $\psi \in L_{p}^{0}$ с рядом Фурье $\sigma(\lambda, f)$, где $\lambda=\left\{\lambda_{n_{1} n_{2}}=n_{1}^{r_{1}} n_{2}^{r_{2}} \gamma_{1}\left(n_{1}\right) \gamma_{2}\left(n_{2}\right)\right\}$, mo $I(f, \tau, p) \ll\|\psi\|_{p}$.

Лемма 2 (см. [6]). Пусть функиия $f \in L_{p}^{0}, 1<p<\infty$, и имеет ряд Фурье (1). Тогда

$$
\|f\|_{p} \asymp\left(\int_{0}^{2 \pi} \int_{0}^{2 \pi}\left\langle\sum_{m_{1}=1}^{\infty} \sum_{m_{1}=1}^{\infty} \Delta_{m_{1} m_{2}}^{2}\right\rangle^{p / 2} d x_{1} d x_{2}\right)^{1 / p} .
$$

ЛЕмма 3 (см. [6]). Пусть функиия $f \in L_{p}^{0}, 1<p<\infty$, и имеет ряд фурье (1), а последовательность $\lambda=\left\{\lambda_{n_{1} n_{2}}\right\}$ такова, ито $\left|\lambda_{n_{1} n_{2}}\right| \leqslant M u$

$$
\begin{gathered}
\sum_{m_{1}=2^{n_{1}-1}}^{2^{n_{1}}-1}\left|\lambda_{m_{1} n_{2}}-\lambda_{m_{1}+1, n_{2}}\right| \leqslant M, \quad \sum_{m_{2}=2^{n_{2}-1}}^{2^{n_{2}}-1}\left|\lambda_{n_{1} m_{2}}-\lambda_{n_{1}, m_{2}+1}\right| \leqslant M, \\
\sum_{m_{1}=2^{n_{1}-1}}^{2^{n_{1}}-1} \sum_{m_{2}=2^{n_{2}-1}}^{2^{n_{2}-1}}\left|\lambda_{m_{1} m_{2}}-\lambda_{m_{1}+1, m_{2}}-\lambda_{m_{1}, m_{2}+1}+\lambda_{m_{1}+1, m_{2}+1}\right| \leqslant M, \\
n_{1}, n_{2}=1,2, \ldots .
\end{gathered}
$$

Тогда ряд $\sum_{n_{1}=1}^{\infty} \sum_{n_{2}=1}^{\infty} \lambda_{n_{1} n_{2}} A_{n_{1} n_{2}}\left(x_{1}, x_{2}\right)$ есть ряд Фурье функиии $\varphi(x) \in L_{p}^{0}$, причем $\|\varphi\|_{p} \leqslant C\|f\|_{p}$, где $C=C(p, M)$.

Лемма 4 (см. [2]). Пусть функиия $f \in L_{p}^{0}, 1<p<\infty$, и имеет ряд Фурье (1).

а) Выполнено

$$
\begin{aligned}
\omega_{\beta_{1} \beta_{2}}\left(f, \frac{1}{m_{1}}, \frac{1}{m_{2}}\right)_{p} \asymp & \sum_{n_{1}=m_{1}+1}^{\infty} \sum_{n_{2}=m_{2}+1}^{\infty} A_{n_{1} n_{2}}\left(x_{1}, x_{2}\right) \|_{p} \\
& +m_{2}^{-\beta_{2}}\left\|\sum_{n_{1}=m_{1}+1}^{\infty} \sum_{n_{2}=1}^{m_{2}} n_{2}^{\beta_{2}} A_{n_{1} n_{2}}\left(x_{1}, x_{2}\right)\right\|_{p} \\
& +m_{1}^{-\beta_{1}}\left\|\sum_{n_{1}=1}^{m_{1}} \sum_{n_{2}=m_{2}+1}^{\infty} n_{1}^{\beta_{1}} A_{n_{1} n_{2}}\left(x_{1}, x_{2}\right)\right\|_{p} \\
& +m_{1}^{-\beta_{1}} m_{2}^{-\beta_{2}}\left\|\sum_{n_{1}=1}^{m_{1}} \sum_{n_{2}=1}^{m_{2}} n_{1}^{\beta_{1}} n_{2}^{\beta_{2}} A_{n_{1} n_{2}}\left(x_{1}, x_{2}\right)\right\|_{p} .
\end{aligned}
$$


b) $E c л u f \in Q M, m o$

$$
\begin{aligned}
\omega_{\beta_{1} \beta_{2}}\left(f, \frac{1}{m_{1}}, \frac{1}{m_{2}}\right)_{p} \asymp & \sum_{n_{1}=m_{1}+1}^{\infty} \sum_{n_{2}=m_{2}+1}^{\infty} b_{n_{1} n_{2}}^{p} n_{1}^{p-2} n_{2}^{p-2} \\
& +m_{2}^{-\beta_{2} p} \sum_{n_{1}=m_{1}+1}^{\infty} \sum_{n_{2}=1}^{m_{2}} b_{n_{1} n_{2}}^{p} n_{1}^{p-2} n_{2}^{\beta_{2} p+p-2} \\
& +m_{1}^{-\beta_{1} p} \sum_{n_{1}=1}^{m_{1}} \sum_{n_{2}=m_{2}+1}^{\infty} b_{n_{1} n_{2}}^{p} n_{1}^{\beta_{1} p+p-2} n_{2}^{p-2} \\
& \left.+m_{1}^{-\beta_{1} p} m_{2}^{-\beta_{2} p} \sum_{n_{1}=1}^{m_{1}} \sum_{n_{2}=1}^{m_{2}} b_{n_{1} n_{2}}^{p} n_{1}^{\beta_{1} p+p-2} n_{2}^{\beta_{2} p+p-2}\right]^{1 / p} .
\end{aligned}
$$

c) $E c л u f \in \mathscr{L}, m o$

$$
\begin{aligned}
& \omega_{\beta_{1} \beta_{2}}\left(f, 2^{-m_{1}}, 2^{-m_{2}}\right)_{p} \asymp\left[\sum_{n_{1}=m_{1}+1}^{\infty} \sum_{n_{2}=m_{2}+1}^{\infty} b_{2^{n_{1}} 2^{n_{2}}}\right. \\
& +2^{-2 m_{2} \beta_{2}} \sum_{n_{1}=m_{1}+1}^{\infty} \sum_{n_{2}=1}^{m_{2}} 2^{2 n_{2} \beta_{2}} b_{2^{n_{1}} 2^{n_{2}}}^{2} \\
& +2^{-2 m_{1} \beta_{1}} \sum_{n_{1}=0}^{m_{1}} \sum_{n_{2}=m_{2}+1}^{\infty} 2^{2 n_{1} \beta_{1}} b_{2^{n_{1}} 2^{n_{2}}}^{2} \\
& \left.+2^{-2\left(m_{1} \beta_{1}+m_{2} \beta_{2}\right)} \sum_{n_{1}=0}^{m_{1}} \sum_{n_{2}=0}^{m_{2}} 2^{2\left(n_{1} \beta_{1}+n_{2} \beta_{2}\right)} b_{2^{n_{1}} 2^{n_{2}}}^{2}\right]^{1 / 2} \text {. }
\end{aligned}
$$

Лемма 5 (см. [6]). Пусть $p \in[1, \infty)$ и пусть функиия $f(u, v)$ определена на измеримом множестве $E=E_{1} \times E_{2} \subset \mathbb{R}_{n}$, әде $x=(u, v), u=\left(x_{1}, \ldots, x_{m}\right)$, $v=\left(x_{m+1}, \ldots, x_{n}\right)$. Тогда

$$
\left\{\int_{E_{1}}\left|\int_{E_{2}} f(u, v) d v\right|^{p} d u\right\}^{1 / p} \leqslant \int_{E_{2}}\left\{\int_{E_{1}}|f(u, v)|^{p} d u\right\}^{1 / p} d v
$$

для тех $f$, для которых правая часть конечна.

Лемма 6 (см. [7]). Пусть положительная функиия $\xi(t)$ слабо колеблющаяся. Тогда

(1) для любого фиксированного отрезка $[a, b], 0<a<b<\infty$, соотношение $\lim _{t \rightarrow \infty}(\xi(\alpha t) / \xi(t))=1$ выполнено равномерно относительно $\alpha \in[a, b] ;$

(2) для любого $\varepsilon>0$ справедливо соотношение $\sum_{\nu=n}^{\infty} \xi(\nu) \nu^{-(1+\varepsilon)} \ll \xi(n) n^{-\varepsilon}$;

(3) для любого $\varepsilon>0$ справедливо соотношение $\sum_{\nu=1}^{n} \xi(\nu) \nu^{\varepsilon-1} \ll \xi(n) n^{\varepsilon}$. 
Лемма 7 (см. [4], [8]). Пусть $\mu(t)$ - слабо колеблющаяся функиия, удовлетворяющая условию $\left(S l_{s}\right)$ и функиия $\gamma(t)$ определена равенством (3). Тогда

(1) функиия $\gamma(t)$ является возрастающей;

(2) функиия $\gamma(t)$ является слабо колеблющейся;

(3) существует натуральное число $N$ такое, что для всех $n>N$ выполнено неравенство $\gamma(n) \ll \mu(n)$.

ЛЕмма 8 (см. [6]). Пусть $\lambda_{1} \geqslant 0, \lambda_{2} \geqslant 0$. Тогда для любого $\theta>0$

$$
C_{1}(\theta)\left(\lambda_{1}^{\theta}+\lambda_{2}^{\theta}\right) \leqslant\left(\lambda_{1}+\lambda_{2}\right)^{\theta} \leqslant C_{2}(\theta)\left(\lambda_{1}^{\theta}+\lambda_{2}^{\theta}\right) .
$$

5. Доказательство теорем. Пункт а) теоремы 1 . Из леммы 1 следует, что существует функция $\varphi \in L_{p}$ с рядом Фурье $\sigma(\lambda, f)$ и справедливо неравенство $\|\varphi\|_{p} \ll I(f, \theta, p)$.

Докажем оценку $A\left(\varphi, \delta_{1}, \delta_{2}, \tau, p\right) \ll B\left(f, \delta_{1}, \delta_{2}, \theta, p\right)$. Рассмотрим $A\left(\varphi, \delta_{1}, \delta_{2}, f, \tau, p\right)$. Выберем натуральные $n_{1}, n_{2}$ такие, что $2^{-n_{i}}<\delta \leqslant 2^{-n_{i}+1}, i=1,2$. Тогда используя свойства смешанного модуля гладкости и свойства слабо колеблющихся функций (см. леммы 6-8), получим

$$
\begin{aligned}
A^{\tau}\left(\varphi, \delta_{1}, \delta_{2}, \tau, p\right) \ll & 2^{-n_{1} \beta_{1} \tau} \gamma_{1}^{\tau}\left(2^{n_{1}}\right) 2^{-n_{2} \beta_{2} \tau} \gamma_{2}^{\tau}\left(2^{n_{2}}\right) \\
& \times \sum_{\nu_{1}=0}^{n_{1}} \sum_{\nu_{2}=0}^{n_{2}} \frac{2^{\nu_{1} \beta_{1} \tau}}{\mu_{1}^{\tau}\left(2^{\nu_{1}}\right)} \frac{2^{\nu_{2} \beta_{2} \tau}}{\mu_{2}^{\tau}\left(2^{\nu_{2}}\right)} \omega_{\beta_{1}, \beta_{2}}^{\tau}\left(\varphi, \frac{1}{2^{\nu_{1}}}, \frac{1}{2^{\nu_{2}}}\right)_{p} \\
& +2^{-n_{1} \beta_{1} \tau} \gamma_{1}^{\tau}\left(2^{n_{1}}\right) \sum_{\nu_{1}=0}^{n_{1}} \frac{2^{\nu_{1} \beta_{1} \tau}}{\mu_{1}^{\tau}\left(2^{\nu_{1}}\right)} \omega_{\beta_{1}, \beta_{2}}^{\tau}\left(\varphi, \frac{1}{2^{\nu_{1}}}, \frac{1}{2^{n_{2}}}\right)_{p} \\
& +2^{-n_{2} \beta_{2} \tau} \gamma_{2}^{\tau}\left(2^{n_{2}}\right) \sum_{\nu_{2}=0}^{n_{2}} \frac{2^{\nu_{2} \beta_{2} \tau}}{\mu_{2}^{\tau}\left(2^{\nu_{2}}\right)} \omega_{\beta_{1}, \beta_{2}}^{\tau}\left(\varphi, \frac{1}{2^{n_{1}}}, \frac{1}{2^{\nu_{2}}}\right)_{p} \\
& +\omega_{\beta_{1}, \beta_{2}}^{\tau}\left(\varphi, \frac{1}{2^{n_{1}}}, \frac{1}{2^{n_{2}}}\right)_{p} \\
=: & J_{1}+J_{2}+J_{3}+J_{4} .
\end{aligned}
$$

Оценим $J_{2}$. Применяя леммы 4 и 8 , получим

$$
\begin{aligned}
J_{2}^{\theta / \tau} \ll & \left(2^{-n_{1} \beta_{1} \tau} \gamma_{1}^{\tau}\left(2^{n_{1}}\right) \sum_{\nu_{1}=0}^{n_{1}} \frac{2^{\nu_{1} \beta_{1} \tau}}{\mu_{1}^{\tau}\left(2^{\nu_{1}}\right)}\right. \\
& \left.\times\left\|\sum_{\xi_{1}=2^{\nu_{1}}+1}^{\infty} \sum_{\xi_{2}=2^{n_{2}}+1}^{\infty} \xi_{1}^{r_{1}} \xi_{2}^{r_{2}} \gamma_{1}\left(\xi_{1}\right) \gamma_{2}\left(\xi_{2}\right) A_{\xi_{1} \xi_{2}}\left(x_{1}, x_{2}\right)\right\|_{p}^{\tau}\right)^{\theta / \tau} \\
& +\left(2^{-n_{1} \beta_{1} \tau} \gamma_{1}^{\tau}\left(2^{n_{1}}\right) \sum_{\nu_{1}=0}^{n_{1}} \frac{1}{\mu_{1}^{\tau}\left(2^{\nu_{1}}\right)}\right. \\
& \left.\quad \times\left\|\sum_{\xi_{1}=1}^{2^{\nu_{1}}} \sum_{\xi_{2}=2^{n_{2}}+1}^{\infty} \xi_{1}^{r_{1}+\beta_{1}} \xi_{2}^{r_{2}} \gamma_{1}\left(\xi_{1}\right) \gamma_{2}\left(\xi_{2}\right) A_{\xi_{1} \xi_{2}}\left(x_{1}, x_{2}\right)\right\|_{p}^{\tau}\right)^{\theta / \tau}
\end{aligned}
$$




$$
\begin{aligned}
& +\left(2^{-n_{1} \beta_{1} \tau} \gamma_{1}^{\tau}\left(2^{n_{1}}\right) 2^{-n_{2} \beta_{2} \tau} \sum_{\nu_{1}=0}^{n_{1}} \frac{2^{\nu_{1} \beta_{1} \tau}}{\mu_{1}^{\tau}\left(2^{\nu_{1}}\right)}\right. \\
& \left.\quad \times\left\|\sum_{\xi_{1}=2^{\nu_{1}}+1}^{\infty} \sum_{\xi_{2}=1}^{2^{n_{2}}} \xi_{1}^{r_{1}} \xi_{2}^{r_{2}+\beta_{2}} \gamma_{1}\left(\xi_{1}\right) \gamma_{2}\left(\xi_{2}\right) A_{\xi_{1} \xi_{2}}\left(x_{1}, x_{2}\right)\right\|_{p}^{\tau}\right)^{\theta / \tau} \\
& +\left(2^{-n_{1} \beta_{1} \tau} \gamma_{1}^{\tau}\left(2^{n_{1}}\right) 2^{-n_{2} \beta_{2} \tau} \sum_{\nu_{1}=0}^{n_{1}} \frac{1}{\mu_{1}^{\tau}\left(2^{\nu_{1}}\right)}\right. \\
& \left.\quad \times\left\|\sum_{\xi_{1}=1}^{2^{\nu_{1}}} \sum_{\xi_{2}=1}^{2^{n_{2}}} \xi_{1}^{r_{1}+\beta_{1}} \xi_{2}^{r_{2}+\beta_{2}} \gamma_{1}\left(\xi_{1}\right) \gamma_{2}\left(\xi_{2}\right) A_{\xi_{1} \xi_{2}}\left(x_{1}, x_{2}\right)\right\|_{p}^{\tau}\right)^{\theta / \tau} \\
& =: J_{21}+J_{22}+J_{23}+J_{24} .
\end{aligned}
$$

Оценим $J_{21}$. По лемме 8

$$
\begin{aligned}
& J_{21} \ll\left(2^{-n_{1} \beta_{1} \tau} \gamma_{1}^{\tau}\left(2^{n_{1}}\right) \sum_{\nu_{1}=0}^{n_{1}} \frac{2^{\nu_{1} \beta_{1} \tau}}{\mu_{1}^{\tau}\left(2^{\nu_{1}}\right)}\right. \\
&\left.\times\left\|\sum_{\xi_{1}=2^{\nu_{1}}+1}^{2^{n_{1}}} \sum_{\xi_{2}=2^{n_{2}}+1}^{\infty} \xi_{1}^{r_{1}} \xi_{2}^{r_{2}} \gamma_{1}\left(\xi_{1}\right) \gamma_{2}\left(\xi_{2}\right) A_{\xi_{1} \xi_{2}}\left(x_{1}, x_{2}\right)\right\|_{p}^{\tau}\right)^{\theta / \tau} \\
&+\left(2^{-n_{1} \beta_{1} \tau} \gamma_{1}^{\tau}\left(2^{n_{1}}\right) \sum_{\nu_{1}=0}^{n_{1}} \frac{2^{\nu_{1} \beta_{1} \tau}}{\mu_{1}^{\tau}\left(2^{\nu_{1}}\right)}\right. \\
&\left.\quad\left\|\sum_{\xi_{1}=2^{n_{1}}+1}^{\infty} \sum_{\xi_{2}=2^{n_{2}}+1}^{\infty} \xi_{1}^{r_{1}} \xi_{2}^{r_{2}} \gamma_{1}\left(\xi_{1}\right) \gamma_{2}\left(\xi_{2}\right) A_{\xi_{1} \xi_{2}}\left(x_{1}, x_{2}\right)\right\|_{p}^{\tau}\right)^{\theta / \tau} \\
&=: J_{211}+J_{212} .
\end{aligned}
$$

Далее будем использовать неравенства (см. лемму 6)

$$
\sum_{\nu_{1}=0}^{n_{1}} \frac{2^{\nu_{1} \beta_{1} \tau}}{\mu_{1}^{\tau}\left(2^{\nu_{1}}\right)} \ll \frac{2^{n_{1} \beta_{1} \tau}}{\gamma_{1}^{\tau}\left(2^{n_{1}}\right)}, \quad 2^{2 \nu_{j} r_{j}} \gamma_{j}^{2}\left(2^{\nu_{j}}\right) \ll\left(\sum_{m_{j}=n_{j}+1}^{\nu_{j}} 2^{m_{j} r_{j} \theta} \gamma_{j}^{\theta}\left(2^{m_{j}}\right)\right)^{2 / \theta}, j=1,2,
$$

и леммы 2,3 :

$$
\begin{aligned}
J_{212} \ll & \left\{\int _ { 0 } ^ { 2 \pi } \int _ { 0 } ^ { 2 \pi } \left(\sum_{\nu_{1}=n_{1}+1}^{\infty} \sum_{\nu_{2}=n_{2}+1}^{\infty} \Delta_{\nu_{1}+1, \nu_{2}+1}^{2}\right.\right. \\
& \left.\left.\times\left\langle\sum_{m_{1}=n_{1}+1}^{\nu_{1}} \sum_{m_{2}=n_{2}+1}^{\nu_{2}} 2^{m_{1} r_{1} \theta} \gamma_{1}^{\theta}\left(2^{m_{1}}\right) 2^{m_{2} r_{2} \theta} \gamma_{2}^{\theta}\left(2^{m_{2}}\right)\right\rangle^{2 / \theta} d x_{1} d x_{2}\right)^{p / 2}\right\}^{\theta / p} .
\end{aligned}
$$

Применяя неравенство Минковского, получим

$$
\begin{aligned}
J_{212} \ll & \left\{\int _ { 0 } ^ { 2 \pi } \int _ { 0 } ^ { 2 \pi } \left(\sum_{m_{1}=n_{1}+1}^{\infty} \sum_{m_{2}=n_{2}+1}^{\infty} 2^{m_{1} r_{1} \theta} \gamma_{1}^{\theta}\left(2^{m_{1}}\right) 2^{m_{2} r_{2} \theta} \gamma_{2}^{\theta}\left(2^{m_{2}}\right)\right.\right. \\
& \left.\left.\times\left\langle\sum_{\nu_{1}=m_{1}}^{\infty} \sum_{\nu_{2}=m_{2}}^{\infty} \Delta_{\nu_{1}+1, \nu_{2}+1}^{2}\right\rangle^{\theta / 2}\right)^{p / \theta} d x_{1} d x_{2}\right\}^{\theta / p}
\end{aligned}
$$




$$
\begin{aligned}
& \ll \sum_{m_{1}=n_{1}+1}^{\infty} \sum_{m_{2}=n_{2}+1}^{\infty} 2^{m_{1} r_{1} \theta} \gamma_{1}^{\theta}\left(2^{m_{1}}\right) 2^{m_{2} r_{2} \theta} \gamma_{2}^{\theta}\left(2^{m_{2}}\right) \\
& \quad \times\left\{\int_{0}^{2 \pi} \int_{0}^{2 \pi}\left(\sum_{\nu_{1}=m_{1}}^{\infty} \sum_{\nu_{2}=m_{2}}^{\infty} \Delta_{\nu_{1}+1, \nu_{2}+1}^{2}\right)^{p / 2} d x_{1} d x_{2}\right\}^{\theta / p} \\
& \ll B^{\theta}\left(f, \delta_{1}, \delta_{2}, \theta, p\right) .
\end{aligned}
$$

Проведя аналогичные рассуждения, оценим $J_{211}$ :

$$
\begin{aligned}
& J_{211} \ll\left\{\frac { \gamma _ { 1 } ^ { \tau } ( 2 ^ { n _ { 1 } } ) } { 2 ^ { n _ { 1 } \beta _ { 1 } \tau } } \sum _ { \nu _ { 1 } = 0 } ^ { n _ { 1 } } \frac { 2 ^ { \nu _ { 1 } \beta _ { 1 } \tau } } { \mu _ { 1 } ^ { \tau } ( 2 ^ { \nu _ { 1 } } ) } \left(\int _ { 0 } ^ { 2 \pi } \int _ { 0 } ^ { 2 \pi } \left[\sum_{m_{1}=\nu_{1}+1}^{n_{1}} \sum_{m_{2}=n_{2}+1}^{\infty} 2^{2 m_{1} r_{1}+2 m_{2} r_{2}}\right.\right.\right. \\
& \left.\left.\left.\times \gamma_{1}^{2}\left(2^{m_{1}}\right) \gamma_{2}^{2}\left(2^{m_{2}}\right) \Delta_{m_{1}+1, m_{2}+1}^{2}\right]^{p / 2} d x_{1} d x_{2}\right)^{\tau / p}\right\}^{\theta / \tau} \\
& \ll \frac{\gamma_{1}^{\theta}\left(2^{n_{1}}\right)}{2^{n_{1} \beta_{1} \theta}}\left\{\int _ { 0 } ^ { 2 \pi } \int _ { 0 } ^ { 2 \pi } \left(\sum _ { \nu _ { 1 } = 0 } ^ { n _ { 1 } } \frac { 2 ^ { \nu _ { 1 } \beta _ { 1 } \tau } } { \mu _ { 1 } ^ { \tau } ( 2 ^ { \nu _ { 1 } } ) } \left[\sum_{m_{1}=\nu_{1}+1}^{n_{1}} \sum_{m_{2}=n_{2}+1}^{\infty} 2^{2 m_{1} r_{1}+2 m_{2} r_{2}}\right.\right.\right. \\
& \left.\left.\left.\times \gamma_{1}^{2}\left(2^{m_{1}}\right) \gamma_{2}^{2}\left(2^{m_{2}}\right) \Delta_{m_{1}+1, m_{2}+1}^{2}\right]^{\tau / 2}\right)^{p / \tau} d x_{1} d x_{2}\right\}^{\theta / p} \\
& \ll \frac{\gamma_{1}^{\theta}\left(2^{n_{1}}\right)}{2^{n_{1} \beta_{1} \theta}}\left\{\int _ { 0 } ^ { 2 \pi } \int _ { 0 } ^ { 2 \pi } \left(\sum _ { m _ { 1 } = 0 } ^ { n _ { 1 } } \left[\sum _ { \nu _ { 1 } = 0 } ^ { m _ { 1 } } \frac { 2 ^ { \nu _ { 1 } \beta _ { 1 } \tau } } { \mu _ { 1 } ^ { \tau } ( 2 ^ { \nu _ { 1 } } ) } \left\langle\sum_{m_{2}=n_{2}+1}^{\infty} 2^{2 m_{1} r_{1}+2 m_{2} r_{2}}\right.\right.\right.\right. \\
& \left.\left.\left.\left.\times \gamma_{1}^{2}\left(2^{m_{1}}\right) \gamma_{2}^{2}\left(2^{m_{2}}\right) \Delta_{m_{1}+1, m_{2}+1}^{2}\right\rangle^{\tau / 2}\right]^{2 / \tau}\right)^{p / 2} d x_{1} d x_{2}\right\}^{\theta / p} \\
& \ll \frac{\gamma_{1}^{\theta}\left(2^{n_{1}}\right)}{2^{n_{1} \beta_{1} \theta}}\left\{\int _ { 0 } ^ { 2 \pi } \int _ { 0 } ^ { 2 \pi } \left(\sum_{m_{1}=0}^{n_{1}} \sum_{m_{2}=n_{2}+1}^{\infty} 2^{2 m_{1}\left(r_{1}+\beta_{1}\right)}\right.\right. \\
& \left.\left.\times \Delta_{m_{1}+1, m_{2}+1}^{2}\left[\sum_{\nu_{2}=n_{2}+1}^{m_{2}} 2^{\nu_{2} r_{2} \theta} \gamma_{2}^{\theta}\left(2^{\nu_{2}}\right)\right]^{2 / \theta}\right)^{p / 2} d x_{1} d x_{2}\right\}^{\theta / p} \\
& \ll \frac{\gamma_{1}^{\theta}\left(2^{n_{1}}\right)}{2^{n_{1} \beta_{1} \theta}}\left\{\int _ { 0 } ^ { 2 \pi } \int _ { 0 } ^ { 2 \pi } \left(\sum _ { \nu _ { 2 } = n _ { 2 } + 1 } ^ { \infty } 2 ^ { 2 \nu _ { 2 } r _ { 2 } } \gamma _ { 2 } ^ { \theta } ( 2 ^ { \nu _ { 2 } } ) \left[\sum_{m_{1}=0}^{n_{1}} \sum_{m_{2}=n_{2}+1}^{\infty} 2^{2 m_{1}\left(r_{1}+\beta_{1}\right)}\right.\right.\right. \\
& \left.\left.\left.\times \Delta_{m_{1}+1, m_{2}+1}^{2}\right]^{\theta / 2}\right)^{p / \theta} d x_{1} d x_{2}\right\}^{\theta / p} \\
& \ll \frac{\gamma_{1}^{\theta}\left(2^{n_{1}}\right)}{2^{n_{1} \beta_{1} \theta}} \sum_{\nu_{2}=n_{2}+1}^{\infty} 2^{\nu_{2} r_{2} \theta} \gamma_{2}^{\theta}\left(2^{\nu_{2}}\right)\left(\int _ { 0 } ^ { 2 \pi } \int _ { 0 } ^ { 2 \pi } \left[\sum_{m_{1}=0}^{n_{1}} \sum_{m_{2}=\nu_{2}+1}^{\infty} 2^{2 m_{1}\left(r_{1}+\beta_{1}\right)}\right.\right. \\
& \left.\left.\times \Delta_{m_{1}+1, m_{2}+1}^{2}\right]^{p / 2} d x_{1} d x_{2}\right)^{\theta / p} \\
& \ll B^{\theta}\left(f, \delta_{1}, \delta_{2}, \theta, p\right) .
\end{aligned}
$$

Объединяя оценки $J_{211}$ и $J_{212}$, получим $J_{21} \ll B^{\theta}\left(f, \delta_{1}, \delta_{2}, \theta, p\right)$. 
Оценим $J_{22}$. Используя леммы $2,3,5$, будем иметь

$$
\begin{aligned}
& J_{22} \ll \frac{\gamma_{1}^{\theta}\left(2^{n_{1}}\right)}{2^{n_{1} \beta_{1} \theta}}\left\{\sum _ { \nu _ { 1 } = 0 } ^ { n _ { 1 } } \frac { 1 } { \mu _ { 1 } ^ { \tau } ( 2 ^ { \nu _ { 1 } } ) } \left(\int _ { 0 } ^ { 2 \pi } \int _ { 0 } ^ { 2 \pi } \left[\sum_{m_{1}=0}^{\nu_{1}} \sum_{m_{2}=n_{2}+1}^{\infty} 2^{2 m_{1}\left(r_{1}+\beta_{1}\right)+2 m_{2} r_{2}}\right.\right.\right. \\
& \left.\left.\left.\times \gamma_{1}^{2}\left(2^{m_{1}}\right) \gamma_{2}^{2}\left(2^{m_{2}}\right) \Delta_{m_{1}+1, m_{2}+1}^{2}\right]^{p / 2} d x_{1} d x_{2}\right)^{\tau / p}\right\}^{\theta / \tau} \\
& \ll \frac{\gamma_{1}^{\theta}\left(2^{n_{1}}\right)}{2^{n_{1} \beta_{1} \theta}}\left\{\int _ { 0 } ^ { 2 \pi } \int _ { 0 } ^ { 2 \pi } \left(\sum _ { \nu _ { 1 } = 0 } ^ { n _ { 1 } } \frac { 1 } { \mu _ { 1 } ^ { \tau } ( 2 ^ { \nu _ { 1 } } ) } \left[\sum_{m_{1}=0}^{\nu_{1}} \sum_{m_{2}=n_{2}+1}^{\infty} 2^{2 m_{1}\left(r_{1}+\beta_{1}\right)+2 m_{2} r_{2}}\right.\right.\right. \\
& \left.\left.\left.\times \gamma_{1}^{2}\left(2^{m_{1}}\right) \gamma_{2}^{2}\left(2^{m_{2}}\right) \Delta_{m_{1}+1, m_{2}+1}^{2}\right]^{\tau / 2}\right)^{p / \tau} d x_{1} d x_{2}\right\}^{\theta / p} \\
& \ll \frac{\gamma_{1}^{\theta}\left(2^{n_{1}}\right)}{2^{n_{1} \beta_{1} \theta}}\left\{\int _ { 0 } ^ { 2 \pi } \int _ { 0 } ^ { 2 \pi } \left(\sum _ { m _ { 1 } = 0 } ^ { n _ { 1 } } \left[\sum _ { \nu _ { 1 } = m _ { 1 } } ^ { n _ { 1 } } \frac { \gamma _ { 1 } ^ { \tau } ( 2 ^ { m _ { 1 } } ) } { \mu _ { 1 } ^ { \tau } ( 2 ^ { \nu _ { 1 } } ) } \left\langle\sum_{m_{2}=n_{2}+1}^{\infty} 2^{2 m_{1}\left(r_{1}+\beta_{1}\right)+2 m_{2} r_{2}}\right.\right.\right.\right. \\
& \left.\left.\left.\left.\times \gamma_{2}^{2}\left(2^{m_{2}}\right) \Delta_{m_{1}+1, m_{2}+1}^{2}\right\rangle^{\tau / 2}\right]^{2 / \tau}\right)^{p / 2} d x_{1} d x_{2}\right\}^{\theta / p} \text {. }
\end{aligned}
$$

Используя свойства функции $\gamma_{1}(t)$, нетрудно проверить, что

$$
\sum_{\nu_{1}=m_{1}}^{n_{1}} \frac{1}{\mu_{1}^{\tau}\left(2^{\nu_{1}}\right)} \ll \frac{1}{\gamma_{1}^{\tau}\left(2^{m_{1}}\right)} .
$$

Тогда (см. оценку $\left.J_{211}\right)$

$$
\begin{aligned}
J_{22} \ll & \frac{\gamma_{1}^{\theta}\left(2^{n_{1}}\right)}{2^{n_{1} \beta_{1} \theta}}\left\{\int _ { 0 } ^ { 2 \pi } \int _ { 0 } ^ { 2 \pi } \left(\sum_{m_{1}=0}^{n_{1}} \sum_{m_{2}=n_{2}+1}^{\infty} 2^{2 m_{1}\left(r_{1}+\beta_{1}\right)}\right.\right. \\
& \left.\left.\times \Delta_{m_{1}+1, m_{2}+1}^{2}\left[\sum_{\nu_{2}=n_{2}+1}^{m_{2}} 2^{\nu_{2} r_{2} \theta} \gamma_{2}^{\theta}\left(2^{\nu_{2}}\right)\right]^{2 / \theta}\right)^{p / 2} d x_{1} d x_{2}\right\}^{\theta / p} \\
\ll & B^{\theta}\left(f, \delta_{1}, \delta_{2}, \theta, p\right) .
\end{aligned}
$$

Оценим $J_{23}$. Используя лемму 8 , получим

$$
\begin{aligned}
J_{23} \ll & \frac{\gamma_{1}^{\theta}\left(2^{n_{1}}\right)}{2^{n_{1} \beta_{1} \theta}} 2^{-n_{2} \beta_{2} \theta}\left(\sum_{\nu_{1}=0}^{n_{1}} \frac{2^{\nu_{1} \beta_{1} \tau}}{\mu_{1}^{\tau}\left(2^{\nu_{1}}\right)} \| \sum_{\xi_{1}=2^{\nu_{1}}+1}^{2^{n_{1}}} \sum_{\xi_{2}=1}^{2^{n_{2}}} \xi_{1}^{r_{1}} \xi_{2}^{r_{2}+\beta_{2}}\right. \\
& \left.\times \gamma_{1}\left(\xi_{1}\right) \gamma_{2}\left(\xi_{2}\right) A_{\xi_{1} \xi_{2}}\left(x_{1}, x_{2}\right) \|_{p}^{\tau}\right)^{\theta / \tau} \\
+ & \frac{\gamma_{1}^{\theta}\left(2^{n_{1}}\right)}{2^{n_{1} \beta_{1} \theta}} 2^{-n_{2} \beta_{2} \theta}\left(\sum_{\nu_{1}=0}^{n_{1}} \frac{2^{\nu_{1} \beta_{1} \tau}}{\mu_{1}^{\tau}\left(2^{\nu_{1}}\right)} \| \sum_{\xi_{1}=2^{n_{1}}+1} \sum_{\xi_{2}=1}^{\infty} \xi_{1}^{r_{1}} \xi_{2}^{r_{2}+\beta_{2}}\right. \\
& \left.\times \gamma_{1}\left(\xi_{1}\right) \gamma_{2}\left(\xi_{2}\right) A_{\xi_{1} \xi_{2}}\left(x_{1}, x_{2}\right) \|_{p}^{\tau}\right)^{\theta / \tau} \\
=: & J_{231}+J_{232} .
\end{aligned}
$$


При оценке $J_{231}$ будем использовать такие же преобразования, как и при оценке $J_{211}$ :

$$
\begin{aligned}
J_{231} \ll & \frac{\gamma_{1}^{\theta}\left(2^{n_{1}}\right)}{2^{n_{1} \beta_{1} \theta}} 2^{-n_{2} \beta_{2} \theta}\left\{\int _ { 0 } ^ { 2 \pi } \int _ { 0 } ^ { 2 \pi } \left(\sum_{m_{1}=0}^{n_{1}} \sum_{m_{2}=0}^{n_{2}} 2^{2 m_{1}\left(r_{1}+\beta_{1}\right)+2 m_{2}\left(r_{2}+\beta_{2}\right)}\right.\right. \\
& \left.\left.\times \gamma_{2}^{2}\left(2^{m_{2}}\right) \Delta_{m_{1}+1, m_{2}+1}^{2}\right)^{p / 2} d x_{1} d x_{2}\right\}^{\theta / p} \\
\ll & \frac{\gamma_{1}^{\theta}\left(2^{n_{1}}\right)}{2^{n_{1} \beta_{1} \theta}} \frac{\gamma_{2}^{\theta}\left(2^{n_{2}}\right)}{2^{n_{2} \beta_{2} \theta}}\left\{\int _ { 0 } ^ { 2 \pi } \int _ { 0 } ^ { 2 \pi } \left(\sum_{m_{1}=0}^{n_{1}} \sum_{m_{2}=0}^{n_{2}} 2^{2 m_{1}\left(r_{1}+\beta_{1}\right)+2 m_{2}\left(r_{2}+\beta_{2}\right)}\right.\right. \\
& \left.\left.\times \Delta_{m_{1}+1, m_{2}+1}^{2}\right)^{p / 2} d x_{1} d x_{2}\right\}^{\theta / p} \\
\ll & B^{\theta}\left(f, \delta_{1}, \delta_{2}, \theta, p\right) .
\end{aligned}
$$

Оценим $J_{232}$ аналогично оценке $J_{212}$ :

$$
\begin{aligned}
J_{232} \ll & 2^{-n_{2} \beta_{2} \theta} \sum_{m_{1}=n_{1}+1}^{\infty} 2^{m_{1} r_{1} \theta} \gamma_{1}^{\theta}\left(2^{m_{1}}\right)\left\{\int _ { 0 } ^ { 2 \pi } \int _ { 0 } ^ { 2 \pi } \left(\sum_{\nu_{1}=m_{1}}^{\infty} \sum_{\nu_{2}=0}^{n_{2}} 2^{2 \nu_{2}\left(r_{2}+\beta_{2}\right)}\right.\right. \\
& \left.\left.\times \gamma_{2}^{2}\left(2^{\nu_{2}}\right) \Delta_{\nu_{1}+1, \nu_{2}+1}^{2}\right)^{p / 2} d x_{1} d x_{2}\right\}^{\theta / p} \\
\ll & \frac{\gamma_{2}^{\theta}\left(2^{n_{2}}\right)}{2^{n_{2} \beta_{2} \theta}} \sum_{m_{1}=n_{1}+1}^{\infty} 2^{m_{1} r_{1} \theta} \gamma_{1}^{\theta}\left(2^{m_{1}}\right)\left\{\int _ { 0 } ^ { 2 \pi } \int _ { 0 } ^ { 2 \pi } \left(\sum_{\nu_{1}=m_{1}}^{\infty} \sum_{\nu_{2}=0}^{n_{2}} 2^{2 \nu_{2}\left(r_{2}+\beta_{2}\right)}\right.\right. \\
& \left.\left.\times \Delta_{\nu_{1}+1, \nu_{2}+1}^{2}\right)^{p / 2} d x_{1} d x_{2}\right\}^{\theta / p} \\
\ll & B^{\theta}\left(f, \delta_{1}, \delta_{2}, \theta, p\right) .
\end{aligned}
$$

Оценим $J_{24}$, используя те же методы, которыми пользовались при оценке $J_{22}$ :

$$
\begin{aligned}
J_{24} \ll & \frac{\gamma_{1}^{\theta}\left(2^{n_{1}}\right)}{2^{n_{1} \beta_{1} \theta}} 2^{-n_{2} \beta_{2} \theta}\left\{\int _ { 0 } ^ { 2 \pi } \int _ { 0 } ^ { 2 \pi } \left(\sum_{m_{1}=0}^{n_{1}} \sum_{m_{2}=0}^{n_{2}} 2^{2 m_{1}\left(r_{1}+\beta_{1}\right)+2 m_{2}\left(r_{2}+\beta_{2}\right)}\right.\right. \\
& \left.\left.\times \gamma_{2}^{2}\left(2^{m_{2}}\right) \Delta_{m_{1}+1, m_{2}}^{2}\right)^{p / 2} d x_{1} d x_{2}\right\}^{\theta / p} \\
\ll & \frac{\gamma_{1}^{\theta}\left(2^{n_{1}}\right)}{2^{n_{1} \beta_{1} \theta}} \frac{\gamma_{2}^{\theta}\left(2^{n_{2}}\right)}{2^{n_{2} \beta_{2} \theta}}\left\{\int _ { 0 } ^ { 2 \pi } \int _ { 0 } ^ { 2 \pi } \left(\sum_{m_{1}=0}^{n_{1}} \sum_{m_{2}=0}^{n_{2}} 2^{2 m_{1}\left(r_{1}+\beta_{1}\right)+2 m_{2}\left(r_{2}+\beta_{2}\right)}\right.\right. \\
& \left.\left.\times \Delta_{m_{1}+1, m_{2}}^{2}\right)^{p / 2} d x_{1} d x_{2}\right\} \\
\ll & B^{\theta}\left(f, \delta_{1}, \delta_{2}, \theta, p\right) .
\end{aligned}
$$

Объединяя полученные оценки $J_{21}, J_{22}, J_{23}, J_{24}$, получим $J_{2}^{\theta / \tau} \ll B^{\theta}\left(f, \delta_{1}, \delta_{2}, \theta, p\right)$. Аналогично получим, что $J_{j}^{\theta / \tau} \ll B^{\theta}\left(f, \delta_{1}, \delta_{2}, \theta, p\right), j=1,3,4$. Таким образом, оценка $A^{\theta}\left(\varphi, \delta_{1}, \delta_{2}, \tau, p\right) \ll B^{\theta}\left(f, \delta_{1}, \delta_{2}, \theta, p\right)$ доказана, что завершает доказательство пункта а). 
Докажем пункт b) теоремы 1. Используя свойства смешанного модуля гладкости и лемму 7 , будем иметь

$$
\begin{aligned}
B^{\theta}\left(f, \delta_{1}, \delta_{2}, \tau, p\right) \ll & \left(\sum_{m_{1}=n_{1}}^{\infty} \sum_{m_{2}=n_{2}}^{\infty} 2^{m_{1} r_{1} \tau} 2^{m_{2} r_{2} \tau} \gamma_{1}^{\tau}\left(2^{m_{1}}\right) \gamma_{2}^{\tau}\left(2^{m_{2}}\right)\right. \\
& \left.\times \omega_{r_{1}+\beta_{1}, r_{2}+\beta_{2}}^{\tau}\left(f, \frac{1}{2^{m_{1}}}, \frac{1}{2^{m_{2}}}\right)_{p}\right)^{\theta / \tau} .
\end{aligned}
$$

Используя леммы 4 и 8 , получим

$$
\begin{aligned}
& B^{\theta}\left(f, \delta_{1}, \delta_{2}, \tau, p\right) \ll\left(\sum_{m_{1}=n_{1}}^{\infty} \sum_{m_{2}=n_{2}}^{\infty} 2^{m_{1} r_{1} \tau} 2^{m_{2} r_{2} \tau} \gamma_{1}^{\tau}\left(2^{m_{1}}\right) \gamma_{2}^{\tau}\left(2^{m_{2}}\right)\right. \\
& \left.\times\left\|\sum_{\xi_{1}=2^{m_{1}}+1}^{\infty} \sum_{\xi_{2}=2^{m_{2}}+1}^{\infty} A_{\xi_{1} \xi_{2}}\left(x_{1}, x_{2}\right)\right\|^{\tau}\right)^{\theta / \tau} \\
& +\left(\sum_{m_{1}=n_{1}}^{\infty} \sum_{m_{2}=n_{2}}^{\infty} 2^{m_{1} r_{1} \tau} 2^{-m_{2} \beta_{2} \tau} \gamma_{1}^{\tau}\left(2^{m_{1}}\right) \gamma_{2}^{\tau}\left(2^{m_{2}}\right)\right. \\
& \left.\times\left\|\sum_{\xi_{1}=2^{m_{1}}+1}^{\infty} \sum_{\xi_{2}=1}^{2^{m_{2}}} \xi_{2}^{r_{2}+\beta_{2}} A_{\xi_{1} \xi_{2}}\left(x_{1}, x_{2}\right)\right\|^{\tau}\right)^{\theta / \tau} \\
& +\left(\sum_{m_{1}=n_{1}}^{\infty} \sum_{m_{2}=n_{2}}^{\infty} 2^{-m_{1} \beta_{1} \tau} 2^{m_{2} r_{2} \tau} \gamma_{1}^{\tau}\left(2^{m_{1}}\right) \gamma_{2}^{\tau}\left(2^{m_{2}}\right)\right. \\
& \left.\times\left\|\sum_{\xi_{1}=1}^{2^{m_{1}}} \sum_{\xi_{2}=2^{m_{2}}+1}^{\infty} \xi_{1}^{r_{1}+\beta_{1}} A_{\xi_{1} \xi_{2}}\left(x_{1}, x_{2}\right)\right\|^{\tau}\right)^{\theta / \tau} \\
& +\left(\sum_{m_{1}=n_{1}}^{\infty} \sum_{m_{2}=n_{2}}^{\infty} 2^{-m_{1} \beta_{1} \tau} 2^{-m_{2} \beta_{1} \tau} \gamma_{1}^{\tau}\left(2^{m_{1}}\right) \gamma_{2}^{\tau}\left(2^{m_{2}}\right)\right. \\
& \left.\times\left\|\sum_{\xi_{1}=1}^{2^{m_{1}}} \sum_{\xi_{2}=1}^{2^{m_{2}}} \xi_{1}^{r_{1}+\beta_{1}} \xi_{2}^{r_{2}+\beta_{2}} A_{\xi_{1} \xi_{2}}\left(x_{1}, x_{2}\right)\right\|^{\tau}\right)^{\theta / \tau} \\
& =: I_{1}+I_{2}+I_{3}+I_{4} \text {. }
\end{aligned}
$$

Оценим $I_{2}$. По лемме 8

$$
\begin{aligned}
I_{2} \ll & \left(\sum_{m_{1}=n_{1}}^{\infty} \sum_{m_{2}=n_{2}}^{\infty} 2^{m_{1} r_{1} \tau} 2^{-m_{2} \beta_{2} \tau} \gamma_{1}^{\tau}\left(2^{m_{1}}\right) \gamma_{2}^{\tau}\left(2^{m_{2}}\right)\right. \\
& \left.\times\left\|\sum_{\xi_{1}=2^{m_{1}}+1}^{\infty} \sum_{\xi_{2}=1}^{2_{2}} \xi_{2}^{r_{2}+\beta_{2}} A_{\xi_{1} \xi_{2}}\left(x_{1}, x_{2}\right)\right\|^{\tau}\right)^{\theta / \tau} \\
& +\left(\sum_{m_{1}=n_{1}}^{\infty} \sum_{m_{2}=n_{2}}^{\infty} 2^{m_{1} r_{1} \tau} 2^{-m_{2} \beta_{2} \tau} \gamma_{1}^{\tau}\left(2^{m_{1}}\right) \gamma_{2}^{\tau}\left(2^{m_{2}}\right)\right. \\
& \left.\times\left\|\sum_{\xi_{1}=2^{m_{1}}+1}^{\infty} \sum_{\xi_{2}=2^{n_{2}}+1}^{\infty} \xi_{2}^{r_{2}+\beta_{2}} A_{\xi_{1} \xi_{2}}\left(x_{1}, x_{2}\right)\right\|^{\tau}\right)^{\theta / \tau} \\
=: & I_{21}+I_{22} .
\end{aligned}
$$


Оценим $I_{21}$. Из лемм 6 и 7 следует, что

$$
\left\langle\sum_{m_{2}=n_{2}}^{\infty} 2^{-m_{2} \beta_{2} \tau} \gamma_{2}^{\tau}\left(2^{m_{2}}\right)\right\rangle^{2 / \tau} \ll 2^{-2 n_{2} \beta_{2}} \gamma_{2}^{2}\left(2^{n_{2}}\right) .
$$

Тогда, применяя леммы 2 и 3 , а затем неравенство Минковского (лемму 5), получим

$$
\begin{aligned}
I_{21} \ll & \frac{\gamma_{2}^{\theta}\left(2^{n_{2}}\right)}{2^{n_{2} \beta_{2} \theta}}\left(\sum _ { m _ { 1 } = n _ { 1 } } ^ { \infty } 2 ^ { m _ { 1 } r _ { 1 } \tau } \gamma _ { 1 } ^ { \tau } ( 2 ^ { m _ { 1 } } ) \left\{\int _ { 0 } ^ { 2 \pi } \int _ { 0 } ^ { 2 \pi } \left\langle\sum_{\nu_{1}=m_{1}}^{\infty} \sum_{\nu_{2}=0}^{n_{2}} 2^{2 \nu_{2}\left(r_{2}+\beta_{2}\right)}\right.\right.\right. \\
& \left.\left.\left.\times \Delta_{\nu_{1}+2, \nu_{2}+1}^{2}\right\rangle^{p / 2} d x_{1} d x_{2}\right\}^{\tau / p}\right)^{\theta / \tau} \\
\ll & \frac{\gamma_{2}^{\theta}\left(2^{n_{2}}\right)}{2^{n_{2} \beta_{2} \theta}}\left(\int _ { 0 } ^ { 2 \pi } \int _ { 0 } ^ { 2 \pi } \left\{\sum _ { \nu _ { 1 } = n _ { 1 } } ^ { \infty } \left[\sum_{m_{1}=n_{1}}^{\nu_{1}} 2^{m_{1} r_{1} \tau}\right.\right.\right. \\
& \left.\left.\left.\times \gamma_{1}^{\tau}\left(2^{m_{1}}\right)\left\langle\sum_{\nu_{2}=0}^{n_{2}} 2^{2 \nu_{2}\left(r_{2}+\beta_{2}\right)} \Delta_{\nu_{1}+2, \nu_{2}+1}^{2}\right\rangle^{\tau / 2}\right]^{2 / \tau}\right\}^{p / 2} d x_{1} d x_{2}\right)^{\theta / p} \\
\ll & \frac{\gamma_{2}^{\theta}\left(2^{n_{2}}\right)}{2^{n_{2} \beta_{2} \theta}}\left(\int _ { 0 } ^ { 2 \pi } \int _ { 0 } ^ { 2 \pi } \left\{\sum_{\nu_{1}=n_{1}}^{\infty} \sum_{\nu_{2}=0}^{n_{2}} 2^{2 \nu_{2}\left(r_{2}+\beta_{2}\right)}\right.\right. \\
& \left.\left.\times \Delta_{\nu_{1}+2, \nu_{2}+1}^{2}\left\langle\sum_{m_{1}=n_{1}}^{\nu_{1}} 2^{m_{1} r_{1} \tau} \gamma_{1}^{\tau}\left(2^{m_{1}}\right)\right\rangle^{2 / \tau}\right\}^{p / 2} d x_{1} d x_{2}\right)^{\theta / p} .
\end{aligned}
$$

Применяя леммы 6 и 7 , получаем справедливость следуюших неравенств:

$$
\begin{gathered}
\left\langle\sum_{m_{1}=n_{1}}^{\nu_{1}} 2^{m_{1} r_{1} \tau} \gamma_{1}^{\tau}\left(2^{m_{1}}\right)\right\rangle^{2 / \tau} \ll 2^{2 \nu_{1} r_{1}} \gamma_{1}^{2}\left(2^{\nu_{1}}\right), \\
1 \ll \frac{\gamma_{2}^{2}\left(2^{\nu_{2}-1}\right)}{\gamma_{2}^{2}\left(2^{n_{2}}\right)}+\gamma_{2}^{2}\left(2^{\nu_{2}-1}\right)\left\langle\sum_{m_{2}=\nu_{2}-1}^{n_{2}} \frac{1}{\mu_{2}^{\theta}\left(2^{m_{2}}\right)}\right\rangle^{2 / \theta} .
\end{gathered}
$$

Тогда

$$
\begin{aligned}
I_{21} \ll & 2^{-n_{2} \beta_{2} \theta}\left(\int _ { 0 } ^ { 2 \pi } \int _ { 0 } ^ { 2 \pi } \left\{\sum_{\nu_{1}=n_{1}}^{\infty} \sum_{\nu_{2}=0}^{n_{2}} 2^{2 \nu_{1} r_{1}} \gamma_{1}^{2}\left(2^{\nu_{1}}\right)\right.\right. \\
& \left.\left.\times 2^{2 \nu_{2}\left(r_{2}+\beta_{2}\right)} \gamma_{2}^{2}\left(2^{\nu_{2}-1}\right) \Delta_{\nu_{1}+2, \nu_{2}+1}^{2}\right\}^{p / 2} d x_{1} d x_{2}\right)^{\theta / p} \\
+ & \frac{\gamma_{2}^{\theta}\left(2^{n_{2}}\right)}{2^{n_{2} \beta_{2} \theta}}\left(\int _ { 0 } ^ { 2 \pi } \int _ { 0 } ^ { 2 \pi } \left\{\sum_{\nu_{1}=n_{1}}^{\infty} \sum_{\nu_{2}=0}^{n_{2}} 2^{2 \nu_{1} r_{1}} \gamma_{1}^{2}\left(2^{\nu_{1}}\right)\right.\right. \\
& \left.\left.\times 2^{2 \nu_{2}\left(r_{2}+\beta_{2}\right)} \gamma_{2}^{2}\left(2^{\nu_{2}-1}\right) \Delta_{\nu_{1}+2, \nu_{2}+1}^{2}\left\langle\sum_{m_{2}=\nu_{2}-1}^{n_{2}} \frac{1}{\mu_{2}^{\theta}\left(2^{m_{2}}\right)}\right\rangle^{2 / \theta}\right\}^{p / 2} d x_{1} d x_{2}\right)^{\theta / p}
\end{aligned}
$$

$$
=: I_{211}+I_{212} \text {. }
$$


Применяя леммы $2-4$, получим

$$
I_{211} \ll \omega_{\beta_{1}, \beta_{2}}^{\theta}\left(\varphi, \frac{1}{2^{n_{1}}}, \frac{1}{2^{n_{2}}}\right)_{p} \ll A^{\theta}\left(\varphi, \delta_{1}, \delta_{2}, \theta, p\right) .
$$

Применяя обобщенное неравенство Минковского (лемма 5), получим

$$
\begin{aligned}
I_{212} \ll & \frac{\gamma_{2}^{\theta}\left(2^{n_{2}}\right)}{2^{n_{2} \beta_{2} \theta}}\left(\int _ { 0 } ^ { 2 \pi } \int _ { 0 } ^ { 2 \pi } \left\{\sum _ { \nu _ { 1 } = n _ { 1 } } ^ { \infty } \left[\sum _ { m _ { 2 } = 0 } ^ { n _ { 2 } } \left\langle\sum_{\nu_{2}=0}^{m_{2}+1} 2^{2 \nu_{1} r_{1}} \gamma_{1}^{2}\left(2^{\nu_{1}}\right)\right.\right.\right.\right. \\
& \left.\left.\left.\left.\times 2^{2 \nu_{2}\left(r_{2}+\beta_{2}\right)} \gamma_{2}^{2}\left(2^{\nu_{2}-1}\right) \frac{\Delta_{\nu_{1}+2, \nu_{2}+1}^{2}}{\mu_{2}^{\theta}\left(2^{m_{2}}\right)}\right\rangle^{\theta / 2}\right]^{2 / \theta}\right\}^{p / 2} d x_{1} d x_{2}\right)^{\theta / p} \\
\ll & \frac{\gamma_{2}^{\theta}\left(2^{n_{2}}\right)}{2^{n_{2} \beta_{2} \theta}}\left(\int _ { 0 } ^ { 2 \pi } \int _ { 0 } ^ { 2 \pi } \left\{\sum _ { m _ { 2 } = 0 } ^ { n _ { 2 } } \left[\sum_{\nu_{1}=n_{1}}^{\infty} \sum_{\nu_{2}=0}^{m_{2}+1} 2^{2 \nu_{1} r_{1}} \gamma_{1}^{2}\left(2^{\nu_{1}}\right)\right.\right.\right. \\
& \left.\left.\left.\times 2^{2 \nu_{2}\left(r_{2}+\beta_{2}\right)} \gamma_{2}^{2}\left(2^{\nu_{2}-1}\right) \frac{\Delta_{\nu_{1}+2, \nu_{2}+1}^{2}}{\mu_{2}^{\theta}\left(2^{m_{2}}\right)}\right]^{2 / \theta}\right\}^{p / \theta} d x_{1} d x_{2}\right)^{\theta / p} \\
\ll & \frac{\gamma_{2}^{\theta}\left(2^{n_{2}}\right)}{2^{n_{2} \beta_{2} \theta}} \sum_{m_{2}=0}^{n_{2}} \frac{1}{\mu_{2}^{\theta}\left(2^{m_{2}}\right)}\left(\int _ { 0 } ^ { 2 \pi } \int _ { 0 } ^ { 2 \pi } \left[\sum_{\nu_{1}=n_{1}}^{\infty} \sum_{\nu_{2}=0}^{m_{2}+1} 2^{2 \nu_{1} r_{1}} \gamma_{1}^{2}\left(2^{\nu_{1}}\right)\right.\right. \\
& \left.\left.\times 2^{2 \nu_{2}\left(r_{2}+\beta_{2}\right)} \gamma_{2}^{2}\left(2^{\nu_{2}-1}\right) \Delta_{\nu_{1}+2, \nu_{2}+1}^{2}\right]^{p / 2} d x_{1} d x_{2}\right)^{\theta / p} .
\end{aligned}
$$

Вновь применяя леммы $2-4$, получим

$$
I_{212} \ll 2^{-n_{2} \beta_{2} \theta} \gamma_{2}^{\theta}\left(2^{n_{2}}\right) \sum_{m_{2}=0}^{n_{2}} \frac{2^{m_{2} \beta_{2} \theta}}{\mu_{2}^{\theta}\left(2^{m_{2}}\right)} \omega_{\beta_{1}, \beta_{2}}^{\theta}\left(\varphi, \frac{1}{2^{n_{1}}}, \frac{1}{2^{m_{2}}}\right)_{p} \ll A^{\theta}\left(\varphi, \delta_{1}, \delta_{2}, \theta, p\right) .
$$

Таким образом, объединяя оценки $I_{211}$ и $I_{212}$, будем иметь $I_{21} \ll A^{\theta}\left(\varphi, \delta_{1}, \delta_{2}, \theta, p\right)$. Оценим $I_{22}$, применяя неравенство Минковского:

$$
\begin{aligned}
I_{22} \ll & \left(\int _ { 0 } ^ { 2 \pi } \int _ { 0 } ^ { 2 \pi } \left\{\sum_{m_{1}=n_{1}}^{\infty} \sum_{m_{2}=n_{2}}^{\infty} 2^{m_{1} r_{1} \tau-m_{2} \beta_{2} \tau} \gamma_{1}^{\tau}\left(2^{m_{1}}\right) \gamma_{2}^{\tau}\left(2^{m_{2}}\right)\right.\right. \\
& \left.\left.\times\left\langle\sum_{\nu_{1}=m_{1}}^{\infty} \sum_{\nu_{2}=n_{2}}^{m_{2}-1} \Delta_{\nu_{1}+2, \nu_{2}+1}^{2} 2^{2 \nu_{2}\left(r_{2}+\beta_{2}\right)}\right\rangle^{\tau / 2}\right\}^{\tau / p} d x_{1} d x_{2}\right)^{\theta / p} \\
\ll & \left(\int _ { 0 } ^ { 2 \pi } \int _ { 0 } ^ { 2 \pi } \left\{\sum_{\nu_{1}=n_{1}}^{\infty} \sum_{\nu_{2}=n_{2}}^{\infty} \Delta_{\nu_{1}+2, \nu_{2}+1}^{2} 2^{2 \nu_{2}\left(r_{2}+\beta_{2}\right)}\right.\right. \\
& \left.\left.\times\left\langle\sum_{m_{1}=n_{1}}^{\nu_{1}} \sum_{m_{2}=\nu_{2}}^{\infty} 2^{m_{1} r_{1} \tau-m_{2} \beta_{2} \tau} \gamma_{1}^{\tau}\left(2^{m_{1}}\right) \gamma_{2}^{\tau}\left(2^{m_{2}}\right)\right\rangle^{2 / \tau}\right\}^{p / 2} d x_{1} d x_{2}\right)^{\theta / p} \\
\ll & \left(\int _ { 0 } ^ { 2 \pi } \int _ { 0 } ^ { 2 \pi } \left\{\sum_{\nu_{1}=n_{1}}^{\infty} \sum_{\nu_{2}=n_{2}}^{\infty} \Delta_{\nu_{1}+2, \nu_{2}+1}^{2}\right.\right. \\
& \left.\left.\times 2^{2 \nu_{1} r_{1}+2 \nu_{2} r_{2}} \gamma_{1}^{2}\left(2^{\nu_{1}}\right) \gamma_{2}^{2}\left(2^{\nu_{2}}\right)\right\}^{p / 2} d x_{1} d x_{2}\right)^{\theta / p} .
\end{aligned}
$$


Применяя леммы 3-5, будем иметь

$$
I_{22} \ll \omega_{\beta_{1}, \beta_{2}}^{\theta}\left(\varphi, \frac{1}{2^{n_{1}}}, \frac{1}{2^{n_{2}}}\right)_{p} \ll A^{\theta}\left(\varphi, \delta_{1}, \delta_{2}, \theta, p\right) .
$$

Таким образом, объединяя оценки для $I_{21}$ и $I_{22}$, получим $I_{2} \ll A^{\theta}\left(\varphi, \delta_{1}, \delta_{2}, \theta, p\right)$. Аналогичным образом доказьваются оценки $I_{j} \ll A^{\theta}\left(\varphi, \delta_{1}, \delta_{2}, \theta, p\right), j=1,3,4$. Таким образом, $B^{\theta}\left(f, \delta_{1}, \delta_{2}, \tau, p\right) \ll A^{\theta}\left(\varphi, \delta_{1}, \delta_{2}, \theta, p\right)$, что завершает доказательство пункта b) теоремы 1.

Теоремы 2 и 3 доказываются теми же методами, что и теорема 1, только вместо пункта а) леммы 4 нужно применять пункты b) и с) соответственно.

6. Заключительные замечания. 1. Все приведенные вьше утверждения для функций двух переменных распространяются на случай $n$ переменных при $n>2$.

2. Для функции $\mu(t)$, рассмотренной в примере 1 , соответствующие оценки в одномерном случае получены в [9].

3. Отметим, что оценки модуля гладкости преобразованного ряда в случае IV отличаются от оценок в случае II только "естественными" отличиями, возникающими в связи с заменой $n_{j}^{-\alpha_{j}}$ на $\ln _{k_{j}}^{-A_{j}}\left(d_{k_{j}} n_{j}\right), j=1,2$ (см. [2] и [3]). Вместе с тем хорошо видно, что полученные оценки для случая V (см. утверждение) отличаются от оценок в случае III (см. [2]) как "естественньми” отличиями, возникающими в связи с заменой $n_{j}^{\alpha_{j}}$ на $\ln _{k_{j}}^{A_{j}}\left(d_{k_{j}} n_{j}\right), j=1,2$, так и числом слагаемых. Существенность этих отличий иллюстрируется ниже примерами 2 и 3.

ПРИмеР 2. Рассмотрим функцию

$$
\begin{gathered}
f_{1}\left(x_{1}, x_{2}\right)=\sum_{n_{1}=1}^{\infty} \sum_{n_{2}=1}^{\infty} \prod_{j=1}^{2}\left(n_{j}^{-\left(r_{j}+\beta_{j}+1-1 / p\right)} \ln ^{-\left(A_{j}+\left(1+\varepsilon_{j}\right) / p\right)}\left(2 n_{j}\right) \cos n_{j} x_{j}\right), \\
\text { где } \quad p \in(1, \infty), \quad r_{j}, \beta_{j}, A_{j}, \varepsilon_{j} \in(0, \infty), \quad j=1,2 .
\end{gathered}
$$

По лемме 4 b)

$$
\omega_{r_{1}+\beta_{1}, r_{2}+\beta_{2}}\left(f_{1}, \frac{1}{m_{1}}, \frac{1}{m_{2}}\right)_{p} \asymp m_{1}^{-\left(r_{1}+\beta_{1}\right)} m_{2}^{-\left(r_{2}+\beta_{2}\right)} .
$$

Если функции $\mu_{j}(t), j=1,2$, определены следующим образом: $\mu_{j}(t)=\ln ^{1 / p+A_{j}}(2 t)$, то они удовлетворяют условию $\left(S l_{p}\right)$ и в этом случае можно рассматривать функции $\gamma_{j}(t)$ $($ см. $(3))$, которые будут записьваться в виде $\gamma_{j}(t)=C_{j} \ln ^{A_{j}}(2 t)$, где $C_{j}$ - постоянные множители. Тогда справедливо неравенство $I\left(f_{1}, p, p\right)<\infty$, и по теореме 2 для случая $\tau=\theta=p$ имеем $B\left(f_{1}, \delta_{1}, \delta_{2}, p, p\right) \asymp A\left(\varphi_{1}, \delta_{1}, \delta_{2}, p, p\right)$, где $\lambda=\left\{\lambda_{n_{1} n_{2}}=n_{1}^{r_{1}} n_{2}^{r_{2}} \times\right.$ $\left.\gamma_{1}\left(n_{1}\right) \gamma_{2}\left(n_{2}\right)\right\}$ и функция $\varphi_{1}$ имеет ряд $\Phi$ урье

$$
\sigma\left(\lambda, f_{1}\right)=\sum_{n_{1}=1}^{\infty} \sum_{n_{2}=1}^{\infty} \prod_{j=1}^{2}\left(n_{j}^{-\left(\beta_{j}+1-1 / p\right)} \ln ^{-\left(1+\varepsilon_{j}\right) / p}\left(2 n_{j}\right) \cos n_{j} x_{j}\right)
$$


Тогда по лемме 4 b)

$$
\omega_{\beta_{1}, \beta_{2}}\left(\varphi_{1}, \frac{1}{m_{1}}, \frac{1}{m_{2}}\right)_{p} \asymp m_{1}^{-\beta_{1}} m_{2}^{-\beta_{2}} .
$$

Несложно показать, что

$$
\begin{aligned}
B^{p}\left(f_{1}, \delta_{1}, \delta_{2}, p, p\right) \asymp & \delta_{1}^{\beta_{1} p} \delta_{2}^{\beta_{2} p} \gamma_{1}^{p}\left(\frac{1}{\delta_{1}}\right) \gamma_{2}^{p}\left(\frac{1}{\delta_{2}}\right) \int_{\delta_{1}}^{1} \int_{\delta_{2}}^{1} \frac{t_{1}^{-\beta_{1} p-1}}{\mu_{1}^{p}\left(\frac{1}{t_{1}}\right)} \frac{t_{2}^{-\beta_{2} p-1}}{\mu_{2}^{p}\left(\frac{1}{t_{2}}\right)} \\
& \times \omega_{\beta_{1} \beta_{2}}^{p}\left(\varphi_{1}, t_{1}, t_{2}\right)_{p} d t_{1} d t_{2} \\
\asymp & \delta_{1}^{\beta_{1} p} \delta_{2}^{\beta_{2} p} \gamma_{1}^{p}\left(\frac{1}{\delta_{1}}\right) \gamma_{2}^{p}\left(\frac{1}{\delta_{2}}\right),
\end{aligned}
$$

но при этом

$$
\begin{aligned}
& \delta_{1}^{\beta_{1} p} \gamma_{1}^{p}\left(\frac{1}{\delta_{1}}\right) \int_{\delta_{1}}^{1} \frac{t_{1}^{-\beta_{1} p-1}}{\mu_{1}^{p}\left(\frac{1}{t_{1}}\right)} \omega_{\beta_{1} \beta_{2}}^{p}\left(\varphi_{1}, t_{1}, \delta_{2}\right)_{p} d t_{1} \asymp \delta_{1}^{\beta_{1} p^{\beta_{2}} \delta_{2} \gamma_{1}^{p}}\left(\frac{1}{\delta_{1}}\right), \\
& \delta_{2}^{\beta_{2} p} \gamma_{2}^{p}\left(\frac{1}{\delta_{2}}\right) \int_{\delta_{2}}^{1} \frac{t_{2}^{-\beta_{2} p-1}}{\mu_{2}^{p}\left(\frac{1}{t_{2}}\right)} \omega_{\beta_{1} \beta_{2}}^{p}\left(\varphi_{1}, \delta_{1}, t_{2}\right)_{p} d t_{2} \asymp \delta_{1}^{\beta_{1} p} \delta_{2}^{\beta_{2} p} \gamma_{2}^{p}\left(\frac{1}{\delta_{2}}\right), \\
& \omega_{\beta_{1} \beta_{2}}^{\tau}\left(\varphi_{1}, \delta_{1}, \delta_{2}\right)_{p} \asymp \delta_{1}^{\beta_{1} p} \delta_{2}^{\beta_{2} p} .
\end{aligned}
$$

ПРИМЕР 3. Рассмотрим функцию

$$
f_{2}\left(x_{1}, x_{2}\right)=\sum_{n_{1}=1}^{\infty} \sum_{n_{2}=1}^{\infty} \prod_{j=1}^{2}\left(n_{j}^{-\left(\beta_{j}+1-1 / p\right)} \cos n_{j} x_{j}\right)
$$

где $p \in(1, \infty), \beta_{j} \in(0, \infty), j=1,2$. Тогда по лемме $\left.4 \mathrm{~b}\right)$

$$
\omega_{r_{1}+\beta_{1}, r_{2}+\beta_{2}}\left(f_{2}, \frac{1}{m_{1}}, \frac{1}{m_{2}}\right)_{p} \asymp m_{1}^{-\beta_{1}} m_{2}^{-\beta_{2}}
$$

где $r_{j}>0$. Следовательно, если функции $\mu_{j}(t)$ определены следующим образом: $\mu_{j}(t)=$ $\ln ^{1 / p+A_{j}}(2 t)$, где $A_{j}>0$, то для любых фиксированных $\beta_{j}>r_{j}$ справедливо неравенство $I\left(f_{2}, p, p\right)<\infty$, где $\gamma_{j}(t)=C_{j} \ln ^{A_{j}}(2 t)$. По теореме 2 для случая $\tau=\theta=p$ имеем $B\left(f_{2}, \delta_{1}, \delta_{2}, p, p\right) \asymp A\left(\varphi_{2}, \delta_{1}, \delta_{2}, p, p\right)$, где $\lambda=\left\{\lambda_{n_{1} n_{2}}=n_{1}^{r_{1}} n_{2}^{r_{2}} \gamma_{1}\left(n_{1}\right) \gamma_{2}\left(n_{2}\right)\right\}$ и функция $\varphi_{2}$ имеет ряд $Ф$ урье

$$
\sigma\left(\lambda, f_{2}\right)=\sum_{n_{1}=1}^{\infty} \sum_{n_{2}=1}^{\infty} \prod_{j=1}^{2}\left(n_{j}^{-\left(\beta_{j}-r_{j}+1-1 / p\right)} \ln ^{A_{j} p}\left(2 n_{j}\right) \cos n_{j} x_{j}\right)
$$

Тогда

$$
\begin{aligned}
B^{p}\left(f_{2}, \delta_{1}, \delta_{2}, p, p\right) & \asymp \omega_{\beta_{1} \beta_{2}}^{p}\left(\varphi_{2}, \delta_{1}, \delta_{2}\right)_{p} \asymp \delta_{1}^{p\left(\beta_{1}-r_{1}\right)} \gamma_{1}^{p}\left(\frac{1}{\delta_{1}}\right) \delta_{2}^{p\left(\beta_{2}-r_{2}\right)} \gamma_{2}^{p}\left(\frac{1}{\delta_{2}}\right) \\
& \asymp \delta_{1}^{p\left(\beta_{1}-r_{1}\right)} \ln ^{A_{1} p} \frac{2}{\delta_{1}} \delta_{2}^{p\left(\beta_{2}-r_{2}\right)} \ln ^{A_{2} p} \frac{2}{\delta_{2}}
\end{aligned}
$$


HO

$$
\begin{aligned}
& \delta_{1}^{\beta_{1} p} \delta_{2}^{\beta_{2} p} \gamma_{1}^{p}\left(\frac{1}{\delta_{1}}\right) \gamma_{2}^{p}\left(\frac{1}{\delta_{2}}\right) \int_{\delta_{1}}^{1} \int_{\delta_{2}}^{1} \frac{t_{1}^{-\beta_{1} p-1}}{\mu_{1}^{p}\left(\frac{1}{t_{1}}\right)} \frac{t_{2}^{-\beta_{2} p-1}}{\mu_{2}^{p}\left(\frac{1}{t_{2}}\right)} \omega_{\beta_{1} \beta_{2}}^{p}\left(\varphi_{2}, t_{1}, t_{2}\right)_{p} d t_{1} d t_{2} \\
& \asymp \prod_{j=1}^{2} \delta_{j}^{p\left(\beta_{j}-r_{j}\right)} \ln ^{A_{j} p-1} \frac{2}{\delta_{j}} \\
& \delta_{1}^{\beta_{1} p} \gamma_{1}^{p}\left(\frac{1}{\delta_{1}}\right) \int_{\delta_{1}}^{1} \frac{t_{1}^{-\beta_{1} p-1}}{\mu_{1}^{p}\left(\frac{1}{t_{1}}\right)} \omega_{\beta_{1} \beta_{2}}^{p}\left(\varphi_{2}, t_{1}, \delta_{2}\right)_{p} d t_{1} \\
& \asymp \delta_{1}^{p\left(\beta_{1}-r_{1}\right)} \ln ^{A_{1} p-1} \frac{2}{\delta_{1}} \delta_{2}^{p\left(\beta_{2}-r_{2}\right)} \ln ^{A_{2} p} \frac{2}{\delta_{2}} \\
& \delta_{2}^{\beta_{2} p} \gamma_{2}^{p}\left(\frac{1}{\delta_{2}}\right) \int_{\delta_{2}}^{1} \frac{t_{2}^{-\beta_{2} p-1}}{\mu_{2}^{p}\left(\frac{1}{t_{2}}\right)} \omega_{\beta_{1} \beta_{2}}^{p}\left(\varphi_{2}, \delta_{1}, t_{2}\right)_{p} d t_{2} \\
& \asymp \delta_{1}^{p\left(\beta_{1}-r_{1}\right)} \ln ^{A_{1} p} \frac{2}{\delta_{1}} \delta_{2}^{p\left(\beta_{2}-r_{2}\right)} \ln ^{A_{2} p-1} \frac{2}{\delta_{2}} .
\end{aligned}
$$

Таким образом, $\omega_{\beta_{1}, \beta_{2}}\left(\varphi, \delta_{1}, \delta_{2}\right)_{p}$ может быть как больше, так и меньше по порядку, чем интегральные слагаемые, входящие в $A\left(\varphi, \delta_{1}, \delta_{2}, p, p\right)$, причем в оценках $B\left(f, \delta_{1}, \delta_{2}\right.$, $p, p) \asymp A\left(\varphi, \delta_{1}, \delta_{2}, p, p\right)$, равно как и в оценках теоремы 1 , главную роль могут играть как те, так и другие слагаемые. В этом и проявляется особенность рассматриваемого случая $\mathrm{V}$.

\section{СПИСОК ЦИТИРОВАННОЙ ЛИТЕРАТУРЫ}

[1] Никольская Н. С. Приближение дифференцируемых функций многих переменных суммами Фурье в метрике $L_{p} / /$ Сиб. матем. ж. 1974. Т. 15. № 2. С. 395-412.

[2] Потапов М.К., Симонов Б. В., Тихонов С. Ю. О классах Бесова, Бесова-Никольского и об оценках смешанных модулей гладкости дробных производных // Тр. МИАН. 2003. T. 243. C. $244-256$.

[3] Потапов М. К., Симонов Б. В. О соотношениях между обобщенньми классами функций Бесова-Никольского и Вейля-Никольского // Тр. МИАН. 1997. Т. 214. С. 250-266.

[4] Тихонов С. Ю. Оценки модулей гладкости преобразованного ряда Фурье // Вестн. МГУ. Сер. 1. Матем., мех. 2002. № 5. С. 58-61.

[5] Потапов М.К. Исследование некоторых классов функций при помощи приближения "углом" // Тр. МИАН. 1972. Т. 117. С. 256-291.

[6] Никольский С. М. Приближение функций многих переменных и теоремы вложения. М.: Наука, 1977.

[7] Сенета Е. Правильно меняющиеся функции. М.: Наука, 1985.

[8] Parameswaran S. Partition functions whose logarithms are slowly oscillating // Trans. Amer. Mat. Soc. 1961. V. 100. P. 217-241.

[9] Tikhonov S. Moduli of smoothness and the interrelation of some classes of functions // Function Spaces. Interpolation Theory and Related Topics. Proc. of the Conf. on Function Spaces, Interpolation Theory And Related Topics in Honour of Jaak Peetre on his 65th Birthday (August 17-22, 2000). Berlin: W. de Gruyter, 2002. P. 413-423.

(М. К. Потапов, С. Ю. Тихонов) Московский государственный

(Б.В. Симонов) Волгоградский технический университет

E-mail: mkpotapov@mail.ru, simonov-b2002@yandex.ru, tikhonov@mccme.ru 